\title{
Inclusive electron-nucleus scattering at high momentum transfer
}

\author{
D. B. Day, J. S. McCarthy, Z. E. Meziani, ${ }^{*}$ R. Minehart, \\ R. Sealock, and S. T. Thornton \\ Department of Physics, University of Virginia Charlottesville, Virginia 22901 \\ J. Jourdan and I. Sick \\ Department of Physics, University of Basel, CH4056 Basel, Switzerland
}

\author{
B. W. Filippone, R. D. McKeown, R. G. Milner, ${ }^{\dagger}$ and D. H. Potterveld ${ }^{\ddagger}$ \\ Kellogg Radiation Laboratory, California Institute of Technology, Pasadena, California 91125
}

Z. Szalata

Department of Physics, American University, Washington, DC, 20016

(Received 11 March 1993; revised manuscript received 14 September 1993)

\begin{abstract}
The response function of nuclei in the quasielastic region at large momentum transfer $(\mathbf{q} \leq 10$ $\mathrm{fm}^{-1}$ ) is measured for a series of nuclei, ${ }^{4} \mathrm{He},{ }^{12} \mathrm{C},{ }^{27} \mathrm{Al},{ }^{56} \mathrm{Fe}$, and ${ }^{197} \mathrm{Au}$, up to large values of the Bjorken scaling variable $x<2.5$.
\end{abstract}

PACS number(s): 25.30.Fj, 13.60.Hb

\section{INTRODUCTION}

The response function of a nucleus as measured by inclusive electron scattering provides important information on the nuclear ground state. This is true in particular if the cross section $\sigma(q, \omega)$ is available over a large range of momentum transfer $q$ and energy transfer $\omega$.

The kinematical region of interest to this paper is the one close to the "quasielastic peak." At an energy loss $\omega$ of the order $\omega_{\mathrm{qe}}=q^{2} / 2 m_{N}$ the inclusive response shows a pronounced peak corresponding to scattering of the electron from a single nucleon which is ejected from the nucleus. This peak has a finite width and a shape that is due to the distribution of the momenta $\mathbf{k}$ and energies $E$ of the initially bound nucleon, described by the spectral function $S(\mathbf{k}, E)$.

While the dominant process at medium energies in inclusive scattering is quasielastic scattering as depicted in Fig. 1, other processes give non-negligible contributions, depending on the kinematics. At an energy loss of 300 $\mathrm{MeV}$ above $\omega_{\text {qe }}$ the excitation of the $\Delta$ resonance appears prominently, and at large $q$ the tail of deep inelastic scattering (DIS) off individual nucleons contributes increasingly.

In the region of the quasielastic peak, $\omega \sim \omega_{\text {qe }}$, the response function yields information on the properties of the bound nucleons. Gross properties of the nu-

\footnotetext{
*Present address: Dept. of Physics, Stanford University, Stanford, CA 94305.

${ }^{\dagger}$ Present address: Dept. of Physics, MIT, Cambridge, MA 02139.

${ }^{\ddagger}$ Present address: Physics Division, Argonne National Laboratory, Argonne, IL 60439.
}

cleon momentum distribution determine the width of the quasielastic peak. Any changes of the nucleon form factor due to the nuclear medium can influence the overall cross section. In the region $\omega \ll \omega_{\text {qe }}$ and at large q the response function is sensitive to the components of the bound-state wave function of high $\mathbf{k}$, of order $\mathbf{q} \sim \mathbf{k}$; studies of nuclei in this region are fundamental to our understanding of short-range nucleon-nucleon correlations. On the low- $\omega$ side of the quasielastic peak one must expect the final-state interaction of the recoiling nucleon to play an important role, particularly at low $\mathbf{q}$ where the energy of the recoiling nucleon is small. Measurement of the response at $\omega>\omega_{\text {qe }}$ can provide information on the nature of nucleon excited states and their change due to the nuclear medium. On the large- $\omega$ side of the quasielastic peak, meson exchange currents (MEC) may also contribute. The study of DIS at very large energy loss $\omega$ shows interesting deviations from the response of free nucleons, and has provided the best signal for a change of the quark wave function of the nucleon due to the nuclear

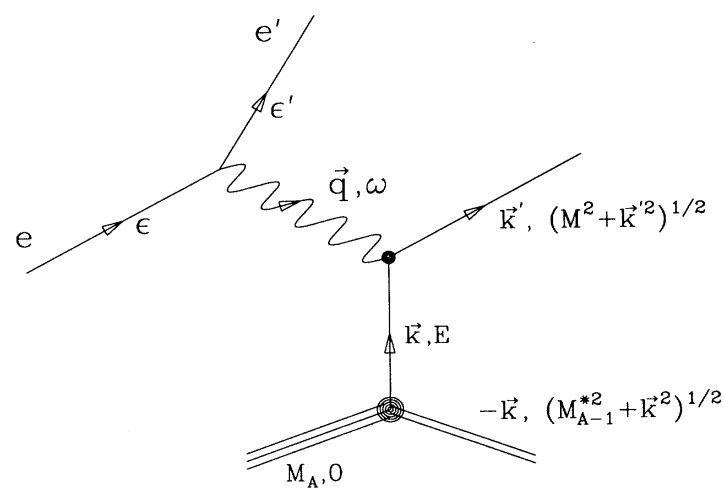

FIG. 1. Quasielastic scattering in impulse approximation. 
medium.

These studies of the inclusive response are particularly interesting if performed at large $\mathbf{q}$. There, the cross section in the $\omega \leq \omega_{\text {qe }}$ region shows approximate scaling in the variable $y$. " $y$ scaling" implies that the cross section $\sigma(q, \omega)$ is no longer a function of the two independent variables $\omega$ and $\mathbf{q}$, but a function of a single variable $y$ that is a function of $\omega$ and $\mathbf{q}$. This phenomenon, and deviations from it, can be exploited to provide a wealth of knowledge on the reaction mechanism, bound nucleon form factors, and selected properties of the nuclear spectral function $S(\mathbf{k}, E)$.

At large $\mathbf{q}$ and small $\omega$ one may also hope to detect scattering from constituents in the nucleus that have a mass larger than the nucleon mass, $m_{N}$. If, with small probability, quarks are deconfined in nuclei, short-range interactions between nucleons could be expected to lead to constituents (of short lifetime) of mass $\sim 2 m_{N}$ identifiable near values of the scaling variable $x=q_{\mu}^{2} / 2 m_{n} \omega \simeq 2$.

At present, very little experimental information on inclusive scattering at large $\mathbf{q}$ is available. While the region of large $\omega$ has been studied by many "EMC"-type experiments $[1,2]$ for $x<0.7$, the region $x>0.7$ has been explored for light nuclei only, $A \leq 3$. For $A=3$ the region of low $\omega$ and large $\mathbf{q}$ has been studied in considerable detail both in view of high-k components, and in connection with $y$ scaling [3].

In this paper, we describe an experiment that studies a series of heavier nuclei, ${ }^{4} \mathrm{He},{ }^{12} \mathrm{C},{ }^{27} \mathrm{Al},{ }^{56} \mathrm{Fe}$, and ${ }^{197} \mathrm{Au}$, and covers the region $\mathbf{q} \leq 10 \mathrm{fm}^{-1}$ of main interest for quasielastic scattering. A brief account of this work has already been given in [4].

With this work we cover a large range of mass numbers of nuclei with approximately equal spacing in $A^{1 / 3}$, to study the evolution of the quasielastic response with the atomic number. From such a series of nuclei one can distinguish effects due to the constant-density nuclear interior from the ones due to the nuclear surface. This allows the extraction of the "nuclear matter" response, a quantity that is of special interest as it is more manageable for quantitative theoretical interpretation than the response of finite, heavy nuclei. Extrapolation from the present data to nuclear matter has already been described in Ref. [5].

The main thrust of this paper is to present the experimental details, the data themselves, and to illustrate some of their features. For a complete description of the experiment, the analysis procedure, and radiative corrections see Ref. [6]. More detailed quantitative interpretation of the results is given elsewhere $[3,7,8]$.

\section{EXPERIMENT}

The experiment was performed using the Nuclear Physics facility at SLAC (NPAS), comprising a special injector, part of the SLAC linear accelerator, and the standard spectrometers located in End Station A.

In order to furnish high-intensity beams of comparatively low energy - several $\mathrm{GeV}$ - a special injector is available at SLAC. This injector provides electrons that are accelerated in the last six sections of the SLAC linear accelerator, thus producing a maximum energy of $\sim$ $5 \mathrm{GeV}$. At the time of this experiment, this facility provided beams up to $4 \mathrm{GeV}$, with 180 pulses per s, $60 \mathrm{~mA}$ peak current and $1.6 \mu$ s duration.

The beam was energy analyzed in the beam line of the $A$ bend. The width of the energy defining slits was set such as to obtain a spread in energy of typically $0.5 \%$ FWHM. Removable screens coated with $\mathrm{ZnS}$ were used to align the beam upstream of the target. Two arrays of thin $\mathrm{Al}$ wires were placed permanently in the beam. The currents on the wires, produced by emission of secondary electrons, were continuously read out using a mini-computer to monitor the beam profile and to keep the beam centered on target by steering it with two magnets under the computer's control.

The beam current was measured pulse by pulse using two nonintercepting toroid transformers, each equipped with two different readout systems. The accuracy achieved for the charge measurement was $<0.5 \%$.

In order to monitor the beam quality, two plastic scintillators were placed upstream and downstream of the target outside the beam line. The upstream scintillator measured the beam halo, and provided a sensitive means to optimize the tuning of the accelerator. The downstream scintillator monitored the beam's pulse shape via the radiation produced by the target.

The target assembly, placed on the spectrometer pivot, carried a liquid-hydrogen $\left(\mathrm{LH}_{2}\right)$ target (pressure $p=2$ atm), a high-pressure ${ }^{4} \mathrm{He}$ gas target $(p=25$ atm) at 20 $\mathrm{K}$, empty dummy targets, and the solid targets for the measurements for nuclei with mass number $A>4$. The arrangement of the targets is shown in Fig. 2.

The $\mathrm{H}_{2}\left({ }^{4} \mathrm{He}\right)$ targets were $15(25) \mathrm{cm}$ in length and had $5 \mathrm{~cm}$ diameter. The end caps had thicknesses of $0.03(0.11) \mathrm{g} / \mathrm{cm}^{2}$ aluminum; the dummy targets were equipped with additional end caps such that the total radiation length was the same as the full target. The liquid (gas) was circulated through the target and heat exchanger $\left(\mathrm{LH}_{2}\right.$ at $p=1$ atm $)$ using a fan, with circulation speeds above $1 \mathrm{~m} / \mathrm{s}$. A cylindrical thin-walled tube placed inside the targets, with the axis aligned with the target cell, ensured that the flow of the liquid (gas) is uniform along the entire length of the target. The target pressures were continuously measured via transducers and the target temperature at both the inlet and outlet was continuously recorded with platinum resistors and hydrogen vapor pressure cells.

The $\mathrm{H}_{2}$ target was run off the saturation point of the liquid by about $2 \mathrm{~K}$, in order to reduce chances for boiling. During the experiment, no change of the density was detected as a function of beam current. The ${ }^{4} \mathrm{He}$ target did show a decrease of density that was linear with the beam current. At the maximum beam intensity employed, this change amounted to $18 \%$.

The solid targets had sizes of typically $3 \times 4 \mathrm{~cm}^{2}$, and thicknesses ranged from 0.9 to $0.37 \mathrm{~g} / \mathrm{cm}^{2}$ depending on mass number. This resulted in a thickness close to $2 \%$ of a radiation length for all targets. For a check of radiative corrections, some runs were taken with an ${ }^{56} \mathrm{Fe}$ target of thickness $6 \%$ of a radiation length. Target thicknesses 


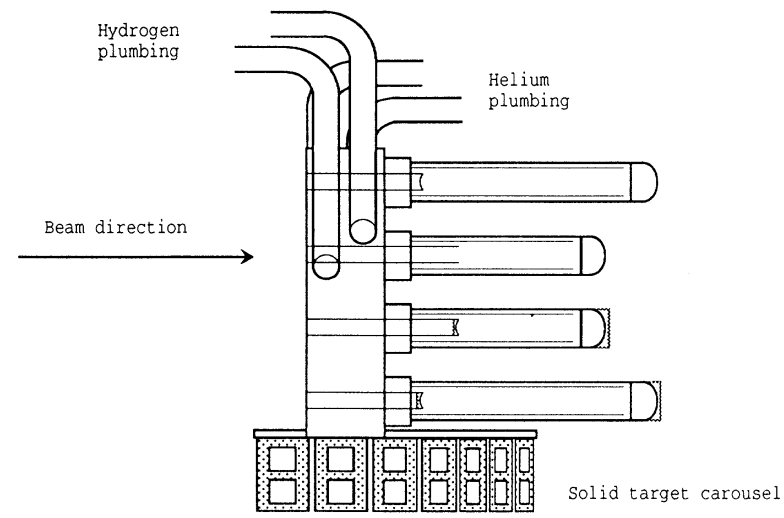

FIG. 2. Arrangement of targets used in the experiment. The assembly can both be moved vertically and rotated around the axis of the carousel carrying the solid targets.

were determined by weight and area, and by mechanical verification of the uniformness; the thicknesses are known to better than $1 \%$. These targets were mounted on a carousel below the cryogenic targets. The target positions were set with digital encoders previously calibrated with the help of a theodolite.

After passing the target, the beam was stopped in the beam dump located outside the end station. The path of the beam downstream of the target was heavily shielded with concrete, in order to stop the radiation produced by electrons multiply scattered in the target.

The scattered electrons were analyzed using the $8 \mathrm{GeV}$ spectrometer. This QQDDQ spectrometer was used in its standard configuration, with $\sim 1 \mathrm{msr}$ solid angle. In this configuration the scattering angle and electron momentum can be reconstructed with good resolution, 0.18 mrad, and $0.13 \%$, respectively. The location of the scattering vertex perpendicular to the spectrometer midplane can be obtained with a resolution of $5 \mathrm{~cm}$. Figure 3 shows a side view of spectrometer and detectors. The scattering angle was calibrated to an accuracy of $\sim 0.002^{\circ}$.

The electrons were detected and identified with a focalplane detector consisting of a gas Cherenkov detector, ten planes of multiwire proportional chambers, a segmented lead glass shower counter ("preradiator") (PR), and a total-absorption (TA) lead glass shower counter.

The Cherenkov detector, $3.1 \mathrm{~m}$ long, operated with nitrogen at 0.7 bar pressure. The resulting index of refraction of 1.0020 allowed discrimination against pions and heavier particles up to $7 \mathrm{GeV} / c$, well above the highest momenta used in the experiment. Ultrarelativistic electrons were detected with an efficiency of 0.999. Additional rejection of unwanted background was provided by the shower counter, which was 20 radiation lengths thick.

The multiwire chambers operated with the "magic gas" mixture. The even-numbered chambers had 176 horizontal wires of $93 \mathrm{~cm}$ length, spaced every $2 \mathrm{~mm}$. The odd-numbered chambers had 480 wires every $2 \mathrm{~mm}$, rotated by $+30^{\circ}$ and $-30^{\circ}$ relative to the vertical direction, respectively. The signals of the $\mathrm{MWPC}$, after amplification and discrimination, were delayed by $450 \mathrm{~ns}$

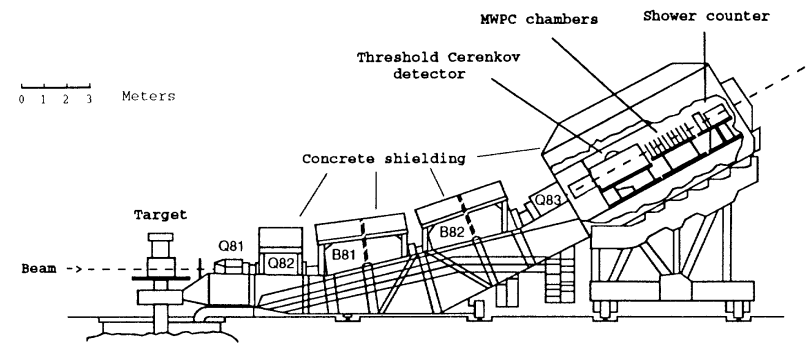

FIG. 3. $8 \mathrm{GeV}$ spectrometer with focal-plane detector system employed: gas Cherenkov counter, 10 planes of MWPC, segmented lead-glass shower counter, and total-absorption lead-glass counter.

and latched into a bit register upon receipt of a fast trigger signal.

The trigger signal was given by a fast coincidence (20 ns) between total absorption counter and preradiator, or the total absorption counter and Cherenkov counter, or both. After a trigger signal, a $12 \mu$ s veto signal inhibited further triggers during the same beam pulse. A random trigger, gated by the downstream beam monitor, was used to check various properties of the electronics at a random moment during the beam pulse.

The number of trigger coincidences was recorded in a scaler, as were the number of coincidences for coincidence time of $40 \mathrm{~ns}$ and $60 \mathrm{~ns}$. The deadtime of the trigger electronics was determined by extrapolating these scalers to a coincidence time of zero.

The information from the detector package was fed into CAMAC ADC's, TDC's, and scalers. This information was read out by a dedicated PDP-11 computer which had the real-time capability needed to achieve the data rate corresponding to the maximum beam pulse frequency of $180 \mathrm{~s}^{-1}$. The data were transmitted to an on-line VAX computer that also read all of the non-time-critical data such as target temperatures and pressures, cumulative toroid values, various beam properties, etc.

The data received by the VAX were stored on magnetic tape. At the same time, the VAX computer performed an on-line analysis of a fraction of the events, to monitor the progress of the experiment.

\section{DATA ANALYSIS}

The reduction of the logtape data to experimental cross section proceeded through three main steps. In the first step, the event-by-event analysis was perfomed, which included the creation of histograms of the data and the reconstruction of the charged-particle trajectories. For each run the good electron events were separated from pion events by requiring that they pass both a Cherenkov and shower-counter pulse height cut. The wire chamber information was used to reconstruct the scattering angles and energy. In addition, events had to pass a track multiplicity cut (when more than one track was found this uniquely identified one track as being due to an electron) and a cut on track position. Only events that passed all four cuts were included in the cross section 
histogram. In the second step the histograms were corrected for the acceptance of the spectrometer, and in the last step the overlapping spectra were summed to yield a complete spectrum for each incident energy, scattering angle, and target. These spectra were then corrected for radiative effects.

The Cherenkov detector and shower counters were found to be highly efficient for the detection of electrons. In the Cherenkov detector, the mean number of photoelectrons was 9 ; with a cut on the pulse height corresponding to two photoelectrons, we found an efficiency of $\mathbf{9 9 . 9 \%}$. The shower counter required a more complicated analysis due to the segmentation of the lead glass. The ADC of each shower-counter segment had its pedestal subtracted and was multiplied by a gain matching coefficient. The PR and TA signals were summed together and normalized to the spectrometer central momentum. This software constructed shower energy gave an energy resolution of $24 \% / \sqrt{E(\mathrm{GeV})}$ FWHM. At the lowest momentum of $1.6 \mathrm{GeV}$, this corresponds to a resolution of $19.0 \%$.

The shower energy spectrum was contaminated by the hadronic shower of pions in the lead glass. The corresponding peak occurred at lower energy because the hadronic shower was not fully contained in the lead glass. By placing a cut on the shower energy, pion rejection was further increased while maintaining an efficiency of $99.9 \%$ for electrons.

In this experiment we did not directly measure the pion rate and pion rejection factor. However, previous experiments [9] with the same setup have measured pion rejection factors of $\geq 10^{4}$. In our worst case the $\pi / e$ ratio was expected to be 10 ; the pion contamination of our final data is thus negligible.

The wire chamber data were used to reconstruct the particle track using the known locations of the wire chambers. The number of tracks per trigger was almost always one, and misidentification was unlikely. If more than one track was found, an attempt was made to uniquely identify one of them as an electron by using the pulse height information in the lead glass block it entered. If only one track satisfied the cut on the pulse height in the lead glass, it was accepted as an electron; any other tracks were discarded.

Once an electron track was uniquely identified, it was checked against a cut on the intersection with the PR lead glass. The purpose of this "fiducial" cut was to reject tracks for which some of the shower energy might leak out of the detector sides because of the lateral spread of the shower.

Events that passed all four cuts were binned in a two-dimensional histogram in $\Delta P / P_{O}$ and $\Delta \theta$, quantities which were derived from the wire chamber information. The resolution of these reconstructed quantities, averaged over spectrometer acceptance, was $0.13 \%$ $\left(\Delta P / P_{O}\right)$ and $0.18 \mathrm{mrad}(\Delta \theta)$. The event histogram spanned $\pm 5.0 \%$ in $\Delta P / P_{O}$ and $\pm 12.0 \mathrm{mrad}$ in $\Delta \theta$.

The number of events in each bin of the $\left(\Delta P / P_{O}, \Delta \theta\right)$ histogram were corrected for the dead time of the data acquisition system (typically less than 4\%) and the detection efficiencies (which consist of the hardware efficiency of the trigger coincidence, the fraction of trigger coincidences logged to tape, and the software cut efficiencies of the data analysis). Cross sections were produced by dividing the corrected number of events in each $\Delta P / P_{O}, \Delta \theta$ bin by the number of scattering centers in the target, the number of incident electrons, and the spectrometer acceptance for that bin.

The dead time was ascertained from information collected from scalers which recorded the number of triggers with various gate widths. The hardware trigger efficiency was $\epsilon_{\text {trig }}=\epsilon_{\mathrm{TA}}\left[1-\left(1-\epsilon_{C}\right)\left(1-\epsilon_{\mathrm{PR}}\right)\right]$ in which $\epsilon_{C}, \epsilon_{\mathrm{PR}}$, and $\epsilon_{\mathrm{TA}}$ are the Cherenkov, PR, and TA hardware efficiencies. The PR lead-glass blocks were relatively thin and they were the least efficient part of the detector package with $\epsilon_{\mathrm{PR}}=0.95$. The Cherenkov hardware efficiency was measured during the checkout as a function of nitrogen gas pressure and phototube voltage in a kinematic region were the $\pi / e$ ratio was low. These data gave $\epsilon_{C}=0.999 \pm 0.001$, with a pion threshold of $7 \mathrm{GeV} / c$. The TA efficiency was $\geq 99.94 \%$ even at the lowest momenta. Together with the PR and Cherenkov efficiencies we had an overall trigger efficiency of $99.94 \%$ for all spectrometer settings.

The wire chambers had individual efficiencies of $95 \%$ on average. The track reconstruction algorithm required only two wire chambers of each type and only five in total in order to find a track so that the efficiency for

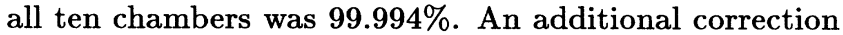
was made for multielectron tracks that satisfied both the Cherenkov and shower energy cuts.

The acceptance of the $8 \mathrm{GeV}$ spectrometer was less than $100 \%$ due to the physical apertures and the fiducial cut mentioned earlier. The physical boundaries of the acceptance function are not sharp but smeared out by multiple scattering. The acceptance function is a function of more than the two variables necessary to calculate the cross section, $\Delta P / P_{O}$, and $\Delta \theta$, so that the correction for the finite acceptance of the spectrometer is an average over the unobserved quantities, weighted by the actual event distribution. The calculation of the correction was done via Monte Carlo techniques, using a TRANSPORT model of the spectrometer optics. The sensitivity of the acceptance function to target thickness, spectrometer angles, and momenta was investigated and no significant variation was found for the solid targets. However, for the extended targets (the $15 \mathrm{~cm} \mathrm{H}$ and the $25 \mathrm{~cm}{ }^{4} \mathrm{He}$ targets) there was a mild angular variation which led us to use separate acceptance functions.

A large body of solid target data was taken in the deep inelastic region (where the cross section is a smooth featureless function of energy loss) in order to test the acceptance function. We found that the average deviation from the acceptance function was $\pm 2 \%$, which was taken as the systematic uncertainty of the acceptance function.

The data on helium and hydrogen contain contributions from the target cell windows. This background was measured by taking data with empty cells which had been outfitted with some additional material to simulate the straggling in the helium and hydrogen target material. The dummy target data passed through the same analysis stream as the other data, and was subtracted from 
the helium and hydrogen event histograms at each spectrometer setting.

The runs at different spectrometer settings for each target were combined to make extended spectra by rebinning the histograms in terms of energy loss $(\omega)$. The bins were chosen to be $15 \mathrm{MeV}$ wide with the center of the lowest bin at $\omega=0$. Bins which overlapped with these $\omega$ bins were summed together, weighted by the fraction of overlap in $\Delta P / P_{O}$. Statistical uncertainties associated with each bin were combined in quadrature.

The hydrogen data were treated slightly differently. Since the position of the elastic peak in $\Delta P / P_{O}$ varied across the $\theta$ acceptance, it was necessary to sum the events at each $\Delta \theta$ bin over a range of $2 \%$ in $\Delta P / P_{O}$ that changed with $\Delta \theta$. After the elastic radiative corrections were made these data were used to check the absolute normalization of the inelastic cross sections.

Before applying the radiative correction (described in the next section) we applied several corrections to the data. These included a correction for the conversion of spectrometer coordinates to the usual polar angles in terms of which we quote the cross sections, and a correction for the finite acceptance of the spectrometer. Both of these contribute such that the effective scattering angle is slightly larger than the spectrometer angle. We quote this corrected angle in the tables given in a later section.

The energy of the spectrometer was calibrated relative to the switchyard by using the positions of the elastic peaks of hydrogen and helium (visible at the three lowest $q^{2}$ 's). The peak positions were compared to those expected from kinematics after taking energy losses in the target into consideration. The largest of these calibration factors amounted to a $0.16 \%$ change in the spectrometer momentum.

The elastic proton data (corrected for radiative effects) was compared to the fit to the world data by Simon et al. [10] as a check of the analysis procedure. Our measured values were typically lower and led us to apply a correction factor of 1.015 to the cross sections.

An additional correction was made for the density changes of the ${ }^{4} \mathrm{He}$ target. These changes from the nominal density were caused by heating by the incident electron beam. The target density as a function of beam current was investigated using data taken at fixed kinematics but with variable beam pulse rates from 10 to 180 $\mathrm{Hz}$. We observed a linear change of the density as a function of beam current, reaching $18 \%$ at the highest power levels.

The systematic errors of target thickness, acceptance function, efficiency corrections, toroid calibration, radiative corrections, and finite binning corrections were added together in quadrature to produce a total systematic uncertainty. These are $3.6 \%, 3.4 \%, 3.4 \%, 3.7 \%$, and 4.0\% for the ${ }^{4} \mathrm{He}, \mathrm{C}, \mathrm{Al}, \mathrm{Fe}$, and $\mathrm{Au}$ targets, respectively.

\section{RADIATIVE CORRECTIONS}

Radiative corrections to the data were made using the formulas of Stein [11], based on the formalism of Mo and
Tsai [12] and Tsai [13]. Application of these corrections to experimental data yields the cross sections which one would observe in the absence of radiative effects.

The incident electron can radiate energy before or after scattering. In the first instance the nuclear interaction is with an electron whose energy is less than the incident beam energy $E_{0}$, and in the latter case the final electron appears at an energy less than it would have otherwise. Consequently, the calculation of radiative corrections before and after scattering requires knowledge of the cross section for all energies between $E_{0}$ and the scattered energy $E^{\prime}$, i.e., cross sections outside of the region covered by our experiment. Fortunately the contribution to the radiative corrections becomes less important as one moves away from the values of $E_{0}$ and $E^{\prime}$ where the correction is being calculated.

To generate the cross sections outside the region of this experiment we constructed an analytic model of the nonradiative cross section. The radiative corrections were calculated in an iterative way in which the parameters of the model are adjusted until the radiated model was consistent with the data. The measured cross sections were then multiplied by the ratio of the cross sections of nonradiative over radiative models.

The model for the inclusive cross section was constructed with the knowledge that in our region of interest, quasielastic scattering dominates; for $q^{2} \geq 1 \mathrm{GeV}^{2}$ and $\omega \geq \frac{q^{2}}{2 m}$ inelastic scattering from nucleons begins to dominate. Both the quasielastic and inelastic processes take place on individual nucleons which have an initial momentum and energy distribution in the ground state. In the impulse approximation we can calculate (ignoring the energy distribution of the nucleons) the inclusive cross sections by a convolution of the electron-nucleon cross section with the momentum distribution $n(k)[14]$. This can be done despite the fact that the momentum distributions are not a directly observable quantity. It is possible because at large momentum transfer the quasielastic cross sections scale [3]-they become independent of $q^{2}$ - and depend on a single variable $y$. The longitudinal momentum distribution, $F(y)=2 \pi \int_{y}^{\infty} n(k) k d k$, can be extracted directly in a region where the cross section is free of any inelastic processes and can be used to predict the quasielastic cross sections at all kinematics. In addition we can use this $n(k)$ to smear the inelastic electronnucleon cross section. While there are some deficiencies in the model just described it serves well in the context of radiative corrections.

The form of the model cross section $\sigma_{\mathrm{nr}}$ was

$$
\sigma_{\mathrm{nr}}=K\left(W^{2}\right)\left(\sigma_{\mathrm{qe}}+\sigma_{\mathrm{dis}}\right)
$$

where $\sigma_{\mathrm{qe}}$ is the quasielastic contribution parametrized using the $y$ scaling model, and $\sigma_{\text {dis }}$ is a parametrized representation of deep inelastic scattering from the nucleon [15] which was smeared with $n(k)$.

We write the quasielastic piece as

$$
\frac{d^{2} \sigma_{\mathrm{qe}}}{d \Omega d E^{\prime}}=\bar{\sigma} F(y)
$$

where $\bar{\sigma}$ is $Z$ times the electron-proton and $N$ times the 
electron-nucleon cross sections [16], evaluated at a momentum of $k=y$ and multiplied by a kinematic factor. Similarly for the inelastic part

$$
\frac{d^{2} \sigma_{\mathrm{dis}}}{d \Omega d E^{\prime}}=\sigma_{\mathrm{Mott}}\left(W_{2}^{A}+2 W_{1}^{A} \tan ^{2} \frac{\theta}{2}\right) .
$$

An estimate of $n(k)$ allows us to calculate the nuclear response functions $W_{1}^{A}$ and $W_{2}^{A}[15]$. Finally, $K\left(W^{2}\right)$ is an arbitrary function of $W^{2}$, the missing mass. $K\left(W^{2}\right)$ was always close to 1 . An initial estimate of $n(k)$ can be extracted from the data before making any radiative corrections and subsequently after each iteration, an improved model for $n(k)$ could be obtained, to be used in the next pass of the correction procedure.

Each spectrum for a given incident energy, angle, and target was unfolded independently. Only data points with an energy loss of at least $50 \mathrm{MeV}$ greater than that of the elastic peak and with statistical uncertainities of less than $50 \%$ were considered. With each iteration the radiative cross section, $\sigma_{\text {rad }}^{i}$, was computed from the model for each energy loss bin $i$. The ratio of the measured cross section to the radiated model, $R_{\sigma}^{i}$, was computed and used to adjust the function $K\left(W^{2}\right)$ to be used in the next iteration. Convergence was achieved if either the radiated model was consistent with the data, or if the radiative corrections were not changing significantly between iterations.

Since the radiative corrections require knowledge of the cross sections outside the region of the data, it is important to ensure that the model for those cross sections does not introduce any model dependence. We found that major variations of the model in the region outside of our data produced insignificant changes in the radiative corrections. This is so because the integrals involved in the corrections are very strongly dominated by the cross section at the point being corrected.

We measured the cross section from solid targets of $2 \%$ and $6 \%$ radiation lengths, in order both to increase the count rate at very small energy transfers and to test the sensitivity of the radiative corrections to the target thickness, as radiative correction factors for the $2 \%$ and $6 \%$ targets are quite different. We found that the ratio of the unfolded cross sections for the thin to thick targets averaged 0.993 , with a $\chi^{2}=1.015$ for 90 data points; this leads us to assign a systematic uncertainty of $2 \%$ to the radiative corrections.

In addition to the radiative corrections for the nuclear targets, we corrected the elastic electron-proton data, a procedure which is straightforward, by comparison. The measured elastic cross section can be written as a multiplicative factor times the unfolded cross section. For these corrections we used Eqs. (II.6) and (II.9) of Mo and Tsai [12]. These equations include effects for recoil and photon emission by the proton, and straggling by the electron before and after the primary scattering. The radiative corrections were done at $2 \mathrm{~mm}$ intervals along the target length (to account for the varying amounts of material before and after the scattering) and the average was applied to our data.

\section{RESULTS}

The cross sections measured in this experiment, corrected for radiative effects, are given in Tables I-VII. The errors quoted there are the random uncertainties only; a systematic error of $3.5 \%$ has to be added. This error includes the uncertainties from the target thickness (1$2 \%$ ), spectrometer acceptance $(2 \%)$, detector efficiency $(1.5 \%)$, toroid calibration $(0.5 \%)$, radiative corrections

TABLE I. Cross sections at $E=2.020 \mathrm{GeV}$ and $\theta=15.02^{\circ}$ as a function of energy loss $\omega(\mathrm{MeV})$. The notation $0.123 \pm 0.456-7$ stands for $(0.123 \pm 0.456) \times 10^{-7} \mathrm{mb} / \mathrm{sr} \mathrm{MeV}$.

\begin{tabular}{cccccc}
\hline \hline$\omega$ & ${ }^{4} \mathrm{He}$ & ${ }^{12} \mathrm{C}$ & ${ }^{27} \mathrm{Al}$ & ${ }^{56} \mathrm{Fe}$ & ${ }^{197} \mathrm{Au}$ \\
\hline 60 & & $0.279 \pm 0.004-4$ & $0.648 \pm 0.012-4$ & $0.128 \pm 0.002-3$ & $0.406 \pm 0.007-3$ \\
75 & & $0.375 \pm 0.005-4$ & $0.838 \pm 0.014-4$ & $0.161 \pm 0.003-3$ & $0.514 \pm 0.008-3$ \\
90 & $0.113 \pm 0.001-4$ & $0.507 \pm 0.011-4$ & $0.101 \pm 0.003-3$ & $0.217 \pm 0.008-3$ & $0.632 \pm 0.020-3$ \\
105 & $0.177 \pm 0.005-4$ & $0.115 \pm 0.003-3$ & $0.232 \pm 0.008-3$ & $0.734 \pm 0.022-3$ \\
120 & $0.229 \pm 0.006-4$ & $0.569 \pm 0.012-4$ & $0.129 \pm 0.002-3$ & $0.246 \pm 0.006-3$ & $0.757 \pm 0.017-3$ \\
135 & $0.289 \pm 0.004-4$ & $0.646 \pm 0.009-4$ & $0.006-3$ & $0.795 \pm 0.017-3$ \\
150 & $0.297 \pm 0.004-4$ & $0.670 \pm 0.010-4$ & $0.133 \pm 0.002-3$ & $0.259 \pm 0.006-3$ \\
165 & $0.290 \pm 0.006-4$ & $0.678 \pm 0.014-4$ & $0.134 \pm 0.003-3$ & $0.271 \pm 0.009-3$ & $0.812 \pm 0.025-3$ \\
180 & $0.248 \pm 0.005-4$ & $0.643 \pm 0.013-4$ & $0.133 \pm 0.003-3$ & $0.257 \pm 0.009-3$ & $0.811 \pm 0.024-3$ \\
195 & $0.201 \pm 0.004-4$ & $0.577 \pm 0.012-4$ & $0.122 \pm 0.003-3$ & $0.243 \pm 0.008-3$ & $0.762 \pm 0.023-3$ \\
210 & $0.166 \pm 0.002-4$ & $0.537 \pm 0.009-4$ & $0.115 \pm 0.002-3$ & $0.237 \pm 0.005-3$ & $0.738 \pm 0.014-3$ \\
225 & $0.132 \pm 0.002-4$ & $0.486 \pm 0.009-4$ & $0.102 \pm 0.002-3$ & $0.216 \pm 0.005-3$ & $0.695 \pm 0.013-3$ \\
240 & $0.106 \pm 0.002-4$ & $0.400 \pm 0.012-4$ & $0.910 \pm 0.030-4$ & $0.199 \pm 0.006-3$ & $0.633 \pm 0.015-3$ \\
255 & $0.881 \pm 0.021-5$ & $0.342 \pm 0.011-4$ & $0.847 \pm 0.029-4$ & $0.176 \pm 0.006-3$ & $0.595 \pm 0.015-3$ \\
270 & $0.751 \pm 0.019-5$ & $0.295 \pm 0.010-4$ & $0.746 \pm 0.027-4$ & $0.165 \pm 0.006-3$ & $0.573 \pm 0.014-3$ \\
285 & $0.681 \pm 0.013-5$ & $0.283 \pm 0.007-4$ & $0.717 \pm 0.019-4$ & $0.150 \pm 0.004-3$ & $0.523 \pm 0.011-3$ \\
300 & $0.675 \pm 0.018-5$ & $0.289 \pm 0.010-4$ & $0.695 \pm 0.026-4$ & $0.161 \pm 0.005-3$ & $0.517 \pm 0.019-3$ \\
315 & $0.672 \pm 0.018-5$ & $0.261 \pm 0.009-4$ & $0.680 \pm 0.025-4$ & $0.142 \pm 0.005-3$ & $0.468 \pm 0.017-3$ \\
330 & $0.721 \pm 0.019-5$ & $0.291 \pm 0.010-4$ & $0.658 \pm 0.025-4$ & $0.133 \pm 0.005-3$ & $0.482 \pm 0.018-3$ \\
345 & $0.773 \pm 0.020-5$ & $0.294 \pm 0.010-4$ & $0.692 \pm 0.026-4$ & $0.140 \pm 0.005-3$ & $0.498 \pm 0.019-3$ \\
360 & $0.817 \pm 0.022-5$ & $0.279 \pm 0.010-4$ & $0.726 \pm 0.028-4$ & $0.139 \pm 0.005-3$ & $0.497 \pm 0.019-3$ \\
\hline \hline
\end{tabular}


TABLE II. Cross sections at $E=2.020 \mathrm{GeV}$ and $\theta=20.02^{\circ}$ as a function of energy loss $\omega(\mathrm{MeV})$. The notation $0.123 \pm 0.456-7$ stands for $(0.123 \pm 0.456) \times 10^{-7} \mathrm{mb} / \mathrm{sr} \mathrm{MeV}$.

\begin{tabular}{|c|c|c|c|c|c|}
\hline$\omega$ & ${ }^{4} \mathrm{He}$ & ${ }^{12} \mathrm{C}$ & ${ }^{27} \mathrm{Al}$ & ${ }^{56} \mathrm{Fe}$ & ${ }^{197} \mathrm{Au}$ \\
\hline 60 & & & $0.644 \pm 0.028-6$ & $0.124 \pm 0.006-5$ & $0.509 \pm 0.026-5$ \\
\hline 75 & & $0.464 \pm 0.014-6$ & $0.130 \pm 0.003-5$ & $0.262 \pm 0.009-5$ & $0.950 \pm 0.035-5$ \\
\hline 90 & & $0.853 \pm 0.019-6$ & $0.234 \pm 0.005-5$ & $0.479 \pm 0.012-5$ & $0.162 \pm 0.004-4$ \\
\hline 105 & & $0.136 \pm 0.002-5$ & $0.353 \pm 0.006-5$ & $0.756 \pm 0.015-5$ & $0.260 \pm 0.005-4$ \\
\hline 120 & $0.281 \pm 0.036-6$ & $0.202 \pm 0.013-5$ & $0.592 \pm 0.034-5$ & $0.116 \pm 0.006-4$ & $0.393 \pm 0.022-4$ \\
\hline 135 & $0.654 \pm 0.050-6$ & $0.334 \pm 0.016-5$ & $0.817 \pm 0.039-5$ & $0.155 \pm 0.007-4$ & $0.554 \pm 0.025-4$ \\
\hline 150 & $0.110 \pm 0.005-5$ & $0.430 \pm 0.015-5$ & $0.106 \pm 0.003-4$ & $0.224 \pm 0.006-4$ & $0.697 \pm 0.022-4$ \\
\hline 165 & $0.161 \pm 0.005-5$ & $0.584 \pm 0.017-5$ & $0.140 \pm 0.003-4$ & $0.269 \pm 0.007-4$ & $0.877 \pm 0.024-4$ \\
\hline 180 & $0.216 \pm 0.005-5$ & $0.763 \pm 0.016-5$ & $0.159 \pm 0.003-4$ & $0.343 \pm 0.007-4$ & $0.102 \pm 0.002-3$ \\
\hline 195 & $0.299 \pm 0.005-5$ & $0.923 \pm 0.018-5$ & $0.191 \pm 0.003-4$ & $0.373 \pm 0.007-4$ & $0.115 \pm 0.002-3$ \\
\hline 210 & $0.389 \pm 0.007-5$ & $0.105 \pm 0.002-4$ & $0.209 \pm 0.005-4$ & $0.416 \pm 0.010-4$ & $0.130 \pm 0.003-3$ \\
\hline 225 & $0.469 \pm 0.007-5$ & $0.112 \pm 0.002-4$ & $0.229 \pm 0.004-4$ & $0.466 \pm 0.009-4$ & $0.139 \pm 0.002-3$ \\
\hline 240 & $0.480 \pm 0.007-5$ & $0.116 \pm 0.002-4$ & $0.240 \pm 0.006-4$ & $0.490 \pm 0.012-4$ & $0.146 \pm 0.003-3$ \\
\hline 255 & $0.508 \pm 0.007-5$ & $0.119 \pm 0.002-4$ & $0.237 \pm 0.004-4$ & $0.516 \pm 0.010-4$ & $0.149 \pm 0.003-3$ \\
\hline 270 & $0.471 \pm 0.009-5$ & $0.117 \pm 0.002-4$ & $0.252 \pm 0.005-4$ & $0.495 \pm 0.011-4$ & $0.148 \pm 0.003-3$ \\
\hline 285 & $0.422 \pm 0.009-5$ & $0.112 \pm 0.002-4$ & $0.245 \pm 0.005-4$ & $0.479 \pm 0.011-4$ & $0.142 \pm 0.003-3$ \\
\hline 300 & $0.354 \pm 0.008-5$ & $0.106 \pm 0.002-4$ & $0.230 \pm 0.005-4$ & $0.449 \pm 0.011-4$ & $0.144 \pm 0.003-3$ \\
\hline 315 & $0.303 \pm 0.012-5$ & $0.970 \pm 0.029-5$ & $0.228 \pm 0.007-4$ & $0.439 \pm 0.014-4$ & $0.146 \pm 0.005-3$ \\
\hline 330 & $0.271 \pm 0.007-5$ & $0.934 \pm 0.020-5$ & $0.215 \pm 0.005-4$ & $0.403 \pm 0.009-4$ & $0.142 \pm 0.003-3$ \\
\hline 345 & $0.238 \pm 0.008-5$ & $0.869 \pm 0.026-5$ & $0.200 \pm 0.006-4$ & $0.432 \pm 0.013-4$ & $0.141 \pm 0.004-3$ \\
\hline 360 & $0.228 \pm 0.007-5$ & $0.832 \pm 0.024-5$ & $0.193 \pm 0.006-4$ & $0.394 \pm 0.012-4$ & $0.131 \pm 0.004-3$ \\
\hline 375 & $0.204 \pm 0.007-5$ & $0.757 \pm 0.022-5$ & $0.184 \pm 0.006-4$ & $0.379 \pm 0.012-4$ & $0.140 \pm 0.004-3$ \\
\hline 390 & $0.188 \pm 0.004-5$ & $0.740 \pm 0.016-5$ & $0.172 \pm 0.003-4$ & $0.358 \pm 0.008-4$ & $0.128 \pm 0.002-3$ \\
\hline 405 & $0.197 \pm 0.004-5$ & $0.706 \pm 0.015-5$ & $0.174 \pm 0.003-4$ & $0.365 \pm 0.008-4$ & $0.125 \pm 0.002-3$ \\
\hline 420 & $0.199 \pm 0.004-5$ & $0.662 \pm 0.021-5$ & $0.172 \pm 0.005-4$ & $0.377 \pm 0.011-4$ & $0.127 \pm 0.003-3$ \\
\hline 435 & $0.206 \pm 0.005-5$ & $0.693 \pm 0.021-5$ & $0.173 \pm 0.005-4$ & $0.336 \pm 0.010-4$ & $0.125 \pm 0.003-3$ \\
\hline 450 & $0.215 \pm 0.005-5$ & $0.760 \pm 0.023-5$ & $0.168 \pm 0.005-4$ & $0.341 \pm 0.011-4$ & $0.126 \pm 0.003-3$ \\
\hline
\end{tabular}

$(2 \%)$, and finite bin correction (1\%).

In Tables I-VII we list only the cross sections for energy loss smaller than that which corresponds to $\Delta$ excitation. For some kinematics and targets the measurements extend much further into the deep inelastic continuum and are available upon request. The cross sections for ${ }^{4} \mathrm{He}$ and ${ }^{56} \mathrm{Fe}$ are shown in Figs. 4 and 5 .

At low $\mathbf{q}$ the data show a clear quasielastic peak, an

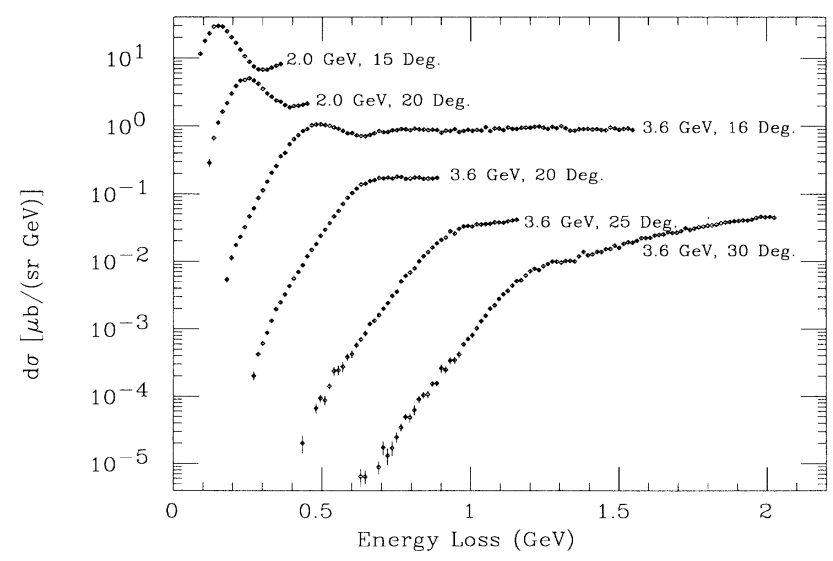

FIG. 4. Cross sections for the ${ }^{4} \mathrm{He}$ nucleus at the various momentum transfers. indication of the $3 / 3$ resonance, and a continuum corresponding to deep inelastic scattering (DIS). With increasing $\mathbf{q}$, the quasielastic peak gets wider, and its separation from the $\Delta$ resonance and DIS becomes poorer. As the target mass increases, the quasielastic peak also gets wider due to the increasing Fermi momentum, with a correspondingly poorer separation from $\Delta$ and DIS. The cross sections in the low- $\omega$ region fall precipitously with

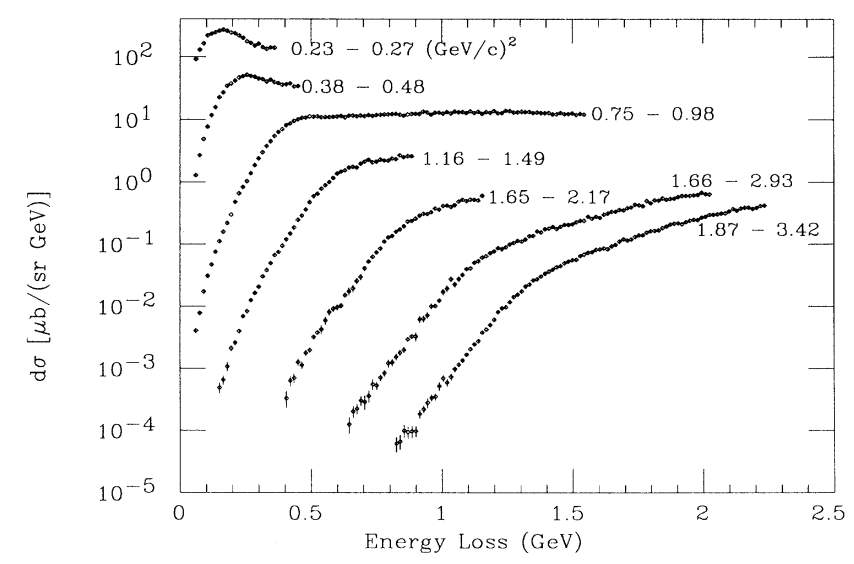

FIG. 5. Cross sections for the iron nucleus at the various momentum transfers. 
increasing $\mathbf{q}$, while the DIS cross section, in accordance with the pointlike nature of the constituents involved, falls only with the Mott cross section.

It is of interest to study the dependence of the cross section on the nuclear mass number $A$. In the region of the quasielastic peak, and at low $\omega$, the cross sections reflect mainly properties of the nuclear spectral function $S(k, E)$, and the $A$ dependence of the cross section gives information on the evolution of $S(k, E)$. As an example, we show in Fig. 6 the cross section per nucleon for 3.595 $\mathrm{GeV}, 16^{\circ}$, and $\omega=180 \mathrm{MeV}$ as a function of $A$. The kinematics correspond to the low- $\omega$ tail of the quasielastic peak. Accordingly, the increasing Fermi momentum for the heavier nuclei leads to a widening of the quasielastic peak and an increase of the cross section. The data presented in Fig. 6 show a rather slow convergence to-

TABLE III. Cross sections at $E=3.595 \mathrm{GeV}$ and $\theta=16.02^{\circ}$ as a function of energy loss $\omega$ (MeV). The notation $0.123 \pm 0.456-7$ stands for $(0.123 \pm 0.456) \times 10^{-7} \mathrm{mb} / \mathrm{sr} \mathrm{MeV}$.

\begin{tabular}{|c|c|c|c|c|c|}
\hline$\omega$ & ${ }^{4} \mathrm{He}$ & ${ }^{12} \mathrm{C}$ & ${ }^{27} \mathrm{Al}$ & ${ }^{56} \mathrm{Fe}$ & ${ }^{197} \mathrm{Au}$ \\
\hline 60 & & & & $0.387 \pm 0.032-8$ & $0.126 \pm 0.013-7$ \\
\hline 75 & & & $0.307 \pm 0.022-8$ & $0.744 \pm 0.045-8$ & $0.260 \pm 0.020-7$ \\
\hline 90 & & & $0.645 \pm 0.031-8$ & $0.165 \pm 0.006-7$ & $0.613 \pm 0.029-7$ \\
\hline 105 & & $0.341 \pm 0.011-8$ & $0.127 \pm 0.004-7$ & $0.294 \pm 0.008-7$ & $0.107 \pm 0.003-6$ \\
\hline 120 & & $0.622 \pm 0.015-8$ & $0.211 \pm 0.005-7$ & $0.447 \pm 0.010-7$ & $0.169 \pm 0.004-6$ \\
\hline 135 & & $0.103 \pm 0.002-7$ & $0.320 \pm 0.007-7$ & $0.718 \pm 0.013-7$ & $0.268 \pm 0.006-6$ \\
\hline 150 & & $0.164 \pm 0.002-7$ & $0.464 \pm 0.008-7$ & $0.106 \pm 0.001-6$ & $0.388 \pm 0.007-6$ \\
\hline 165 & & $0.248 \pm 0.003-7$ & $0.707 \pm 0.010-7$ & $0.150 \pm 0.001-6$ & $0.537 \pm 0.008-6$ \\
\hline 180 & $0.513 \pm 0.046-8$ & $0.366 \pm 0.003-7$ & $0.953 \pm 0.012-7$ & $0.208 \pm 0.002-6$ & $0.738 \pm 0.010-6$ \\
\hline 195 & $0.108 \pm 0.009-7$ & $0.520 \pm 0.004-7$ & $0.140 \pm 0.001-6$ & $0.286 \pm 0.002-6$ & $0.101 \pm 0.001-5$ \\
\hline 210 & $0.167 \pm 0.011-7$ & $0.798 \pm 0.042-7$ & $0.206 \pm 0.008-6$ & $0.464 \pm 0.014-6$ & $0.150 \pm 0.006-5$ \\
\hline 225 & $0.222 \pm 0.012-7$ & $0.115 \pm 0.004-6$ & $0.286 \pm 0.009-6$ & $0.636 \pm 0.016-6$ & $0.194 \pm 0.007-5$ \\
\hline 240 & $0.310 \pm 0.014-7$ & $0.162 \pm 0.005-6$ & $0.370 \pm 0.011-6$ & $0.807 \pm 0.019-6$ & $0.255 \pm 0.008-5$ \\
\hline 255 & $0.447 \pm 0.018-7$ & $0.215 \pm 0.006-6$ & $0.522 \pm 0.013-6$ & $0.100 \pm 0.002-5$ & $0.351 \pm 0.010-5$ \\
\hline 270 & $0.592 \pm 0.021-7$ & $0.268 \pm 0.007-6$ & $0.698 \pm 0.016-6$ & $0.134 \pm 0.002-5$ & $0.454 \pm 0.012-5$ \\
\hline 285 & $0.841 \pm 0.024-7$ & $0.362 \pm 0.008-6$ & $0.835 \pm 0.017-6$ & $0.182 \pm 0.002-5$ & $0.600 \pm 0.013-5$ \\
\hline 300 & $0.109 \pm 0.002-6$ & $0.470 \pm 0.009-6$ & $0.115 \pm 0.002-5$ & $0.234 \pm 0.003-5$ & $0.767 \pm 0.014-5$ \\
\hline 315 & $0.147 \pm 0.003-6$ & $0.610 \pm 0.010-6$ & $0.143 \pm 0.002-5$ & $0.294 \pm 0.003-5$ & $0.948 \pm 0.015-5$ \\
\hline 330 & $0.198 \pm 0.012-6$ & $0.787 \pm 0.030-6$ & $0.176 \pm 0.009-5$ & $0.372 \pm 0.011-5$ & $0.121 \pm 0.005-4$ \\
\hline 345 & $0.250 \pm 0.013-6$ & $0.984 \pm 0.033-6$ & $0.194 \pm 0.010-5$ & $0.442 \pm 0.012-5$ & $0.151 \pm 0.005-4$ \\
\hline 360 & $0.351 \pm 0.015-6$ & $0.121 \pm 0.003-5$ & $0.280 \pm 0.012-5$ & $0.538 \pm 0.013-5$ & $0.178 \pm 0.006-4$ \\
\hline 375 & $0.394 \pm 0.016-6$ & $0.142 \pm 0.004-5$ & $0.330 \pm 0.013-5$ & $0.624 \pm 0.014-5$ & $0.199 \pm 0.006-4$ \\
\hline 390 & $0.534 \pm 0.019-6$ & $0.159 \pm 0.004-5$ & $0.371 \pm 0.013-5$ & $0.692 \pm 0.015-5$ & $0.221 \pm 0.006-4$ \\
\hline 405 & $0.637 \pm 0.016-6$ & $0.186 \pm 0.003-5$ & $0.419 \pm 0.010-5$ & $0.806 \pm 0.013-5$ & $0.246 \pm 0.005-4$ \\
\hline 420 & $0.732 \pm 0.017-6$ & $0.212 \pm 0.004-5$ & $0.443 \pm 0.011-5$ & $0.859 \pm 0.013-5$ & $0.278 \pm 0.005-4$ \\
\hline 435 & $0.845 \pm 0.018-6$ & $0.228 \pm 0.004-5$ & $0.465 \pm 0.011-5$ & $0.949 \pm 0.013-5$ & $0.294 \pm 0.005-4$ \\
\hline 450 & $0.906 \pm 0.026-6$ & $0.231 \pm 0.006-5$ & $0.534 \pm 0.015-5$ & $0.100 \pm 0.002-4$ & $0.312 \pm 0.007-4$ \\
\hline 465 & $0.103 \pm 0.002-5$ & $0.253 \pm 0.006-5$ & $0.552 \pm 0.015-5$ & $0.106 \pm 0.002-4$ & $0.333 \pm 0.007-4$ \\
\hline 480 & $0.106 \pm 0.002-5$ & $0.264 \pm 0.006-5$ & $0.544 \pm 0.014-5$ & $0.107 \pm 0.002-4$ & $0.343 \pm 0.007-4$ \\
\hline 495 & $0.107 \pm 0.002-5$ & $0.265 \pm 0.006-5$ & $0.544 \pm 0.014-5$ & $0.113 \pm 0.002-4$ & $0.354 \pm 0.007-4$ \\
\hline 510 & $0.104 \pm 0.002-5$ & $0.273 \pm 0.006-5$ & $0.533 \pm 0.014-5$ & $0.111 \pm 0.002-4$ & $0.361 \pm 0.007-4$ \\
\hline 525 & $0.102 \pm 0.002-5$ & $0.260 \pm 0.004-5$ & $0.558 \pm 0.012-5$ & $0.112 \pm 0.001-4$ & $0.365 \pm 0.006-4$ \\
\hline 540 & $0.961 \pm 0.019-6$ & $0.265 \pm 0.004-5$ & $0.561 \pm 0.012-5$ & $0.109 \pm 0.001-4$ & $0.348 \pm 0.006-4$ \\
\hline 555 & $0.918 \pm 0.018-6$ & $0.257 \pm 0.004-5$ & $0.570 \pm 0.012-5$ & $0.109 \pm 0.001-4$ & $0.359 \pm 0.006-4$ \\
\hline 570 & $0.855 \pm 0.028-6$ & $0.260 \pm 0.007-5$ & $0.560 \pm 0.019-5$ & $0.109 \pm 0.002-4$ & $0.365 \pm 0.015-4$ \\
\hline 585 & $0.786 \pm 0.027-6$ & $0.242 \pm 0.007-5$ & $0.526 \pm 0.018-5$ & $0.111 \pm 0.002-4$ & $0.373 \pm 0.015-4$ \\
\hline 600 & $0.790 \pm 0.026-6$ & $0.242 \pm 0.006-5$ & $0.534 \pm 0.018-5$ & $0.111 \pm 0.002-4$ & $0.380 \pm 0.015-4$ \\
\hline 615 & $0.730 \pm 0.024-6$ & $0.250 \pm 0.006-5$ & $0.565 \pm 0.018-5$ & $0.113 \pm 0.002-4$ & $0.377 \pm 0.015-4$ \\
\hline 630 & $0.715 \pm 0.024-6$ & $0.240 \pm 0.006-5$ & $0.531 \pm 0.017-5$ & $0.108 \pm 0.002-4$ & $0.380 \pm 0.015-4$ \\
\hline 645 & $0.714 \pm 0.021-6$ & $0.231 \pm 0.005-5$ & $0.533 \pm 0.014-5$ & $0.115 \pm 0.002-4$ & $0.365 \pm 0.011-4$ \\
\hline 660 & $0.743 \pm 0.022-6$ & $0.243 \pm 0.005-5$ & $0.560 \pm 0.014-5$ & $0.115 \pm 0.002-4$ & $0.388 \pm 0.011-4$ \\
\hline 675 & $0.789 \pm 0.022-6$ & $0.242 \pm 0.005-5$ & $0.572 \pm 0.014-5$ & $0.113 \pm 0.002-4$ & $0.399 \pm 0.011-4$ \\
\hline 690 & $0.847 \pm 0.040-6$ & $0.255 \pm 0.009-5$ & $0.555 \pm 0.020-5$ & $0.116 \pm 0.002-4$ & $0.433 \pm 0.015-4$ \\
\hline 705 & $0.818 \pm 0.040-6$ & $0.249 \pm 0.009-5$ & $0.562 \pm 0.021-5$ & $0.113 \pm 0.002-4$ & $0.409 \pm 0.015-4$ \\
\hline 720 & $0.823 \pm 0.040-6$ & $0.273 \pm 0.009-5$ & $0.582 \pm 0.021-5$ & $0.117 \pm 0.002-4$ & $0.430 \pm 0.015-4$ \\
\hline 735 & $0.870 \pm 0.039-6$ & $0.263 \pm 0.009-5$ & $0.601 \pm 0.020-5$ & $0.114 \pm 0.002-4$ & $0.414 \pm 0.015-4$ \\
\hline 750 & $0.875 \pm 0.041-6$ & $0.276 \pm 0.009-5$ & $0.592 \pm 0.021-5$ & $0.117 \pm 0.002-4$ & $0.389 \pm 0.015-4$ \\
\hline 765 & $0.911 \pm 0.021-6$ & $0.274 \pm 0.007-5$ & $0.632 \pm 0.016-5$ & $0.119 \pm 0.002-4$ & $0.428 \pm 0.011-4$ \\
\hline
\end{tabular}


wards the nuclear matter value expected for infinite $A$.

The slow convergence to $A=\infty$ results from the fact that the fraction of nucleons in the central, constantdensity (nuclear matter) region of the nucleus increases very slowly with $A$. Even for a heavy nucleus such as
${ }^{197} \mathrm{Au}$ more than $50 \%$ of the nucleons are still in the surface region of the nucleus, where the density is smaller than the nuclear matter value.

The dependence of the cross section per nucleon on $A$ is better understood if the data are plotted as a function

TABLE IV. Cross sections at $E=3.595 \mathrm{GeV}$ and $\theta=20.02^{\circ}$ as a function of energy loss $\omega$ (MeV). The notation $0.123 \pm 0.456-7$ stands for $(0.123 \pm 0.456) \times 10^{-7} \mathrm{mb} / \mathrm{sr} \mathrm{MeV}$.

\begin{tabular}{|c|c|c|c|c|c|}
\hline$\omega$ & ${ }^{4} \mathrm{He}$ & ${ }^{12} \mathrm{C}$ & ${ }^{27} \mathrm{Al}$ & ${ }^{56} \mathrm{Fe}$ & ${ }^{197} \mathrm{Au}$ \\
\hline 135 & & & & $0.113 \pm 0.053-9$ & \\
\hline 150 & & & & $0.471 \pm 0.091-9$ & \\
\hline 165 & & $0.595 \pm 0.284-10$ & $0.242 \pm 0.095-9$ & $0.633 \pm 0.106-9$ & $0.186 \pm 0.065-8$ \\
\hline 180 & & $0.143 \pm 0.036-9$ & $0.798 \pm 0.164-9$ & $0.104 \pm 0.016-8$ & $0.275 \pm 0.066-8$ \\
\hline 195 & & $0.219 \pm 0.040-9$ & $0.498 \pm 0.117-9$ & $0.203 \pm 0.021-8$ & $0.581 \pm 0.095-8$ \\
\hline 210 & & $0.353 \pm 0.049-9$ & $0.104 \pm 0.016-8$ & $0.246 \pm 0.023-8$ & $0.807 \pm 0.109-8$ \\
\hline 225 & & $0.597 \pm 0.065-9$ & $0.182 \pm 0.021-8$ & $0.386 \pm 0.028-8$ & $0.146 \pm 0.014-7$ \\
\hline 240 & & $0.102 \pm 0.008-8$ & $0.231 \pm 0.025-8$ & $0.665 \pm 0.038-8$ & $0.211 \pm 0.017-7$ \\
\hline 255 & $0.135 \pm 0.057-9$ & $0.124 \pm 0.010-8$ & $0.350 \pm 0.033-8$ & $0.800 \pm 0.043-8$ & $0.318 \pm 0.023-7$ \\
\hline 270 & $0.193 \pm 0.027-9$ & $0.192 \pm 0.011-8$ & $0.527 \pm 0.037-8$ & $0.121 \pm 0.004-7$ & $0.384 \pm 0.019-7$ \\
\hline 285 & $0.405 \pm 0.033-9$ & $0.265 \pm 0.012-8$ & $0.718 \pm 0.042-8$ & $0.158 \pm 0.005-7$ & $0.535 \pm 0.023-7$ \\
\hline 300 & $0.588 \pm 0.040-9$ & $0.376 \pm 0.015-8$ & $0.957 \pm 0.047-8$ & $0.200 \pm 0.005-7$ & $0.691 \pm 0.025-7$ \\
\hline 315 & $0.850 \pm 0.047-9$ & $0.500 \pm 0.038-8$ & $0.133 \pm 0.013-7$ & $0.293 \pm 0.013-7$ & $0.996 \pm 0.048-7$ \\
\hline 330 & $0.127 \pm 0.005-8$ & $0.671 \pm 0.043-8$ & $0.185 \pm 0.015-7$ & $0.370 \pm 0.015-7$ & $0.123 \pm 0.005-6$ \\
\hline 345 & $0.190 \pm 0.006-8$ & $0.844 \pm 0.049-8$ & $0.256 \pm 0.018-7$ & $0.480 \pm 0.017-7$ & $0.163 \pm 0.006-6$ \\
\hline 360 & $0.241 \pm 0.007-8$ & $0.117 \pm 0.005-7$ & $0.305 \pm 0.019-7$ & $0.648 \pm 0.019-7$ & $0.204 \pm 0.006-6$ \\
\hline 375 & $0.316 \pm 0.008-8$ & $0.144 \pm 0.006-7$ & $0.416 \pm 0.023-7$ & $0.724 \pm 0.020-7$ & $0.250 \pm 0.007-6$ \\
\hline 390 & $0.421 \pm 0.009-8$ & $0.178 \pm 0.007-7$ & $0.483 \pm 0.025-7$ & $0.915 \pm 0.023-7$ & $0.316 \pm 0.008-6$ \\
\hline 405 & $0.543 \pm 0.010-8$ & $0.250 \pm 0.007-7$ & $0.554 \pm 0.023-7$ & $0.116 \pm 0.002-6$ & $0.408 \pm 0.009-6$ \\
\hline 420 & $0.680 \pm 0.012-8$ & $0.303 \pm 0.008-7$ & $0.715 \pm 0.026-7$ & $0.144 \pm 0.002-6$ & $0.496 \pm 0.010-6$ \\
\hline 435 & $0.858 \pm 0.014-8$ & $0.399 \pm 0.010-7$ & $0.906 \pm 0.029-7$ & $0.181 \pm 0.003-6$ & $0.612 \pm 0.011-6$ \\
\hline 450 & $0.116 \pm 0.006-7$ & $0.469 \pm 0.023-7$ & $0.116 \pm 0.005-6$ & $0.240 \pm 0.011-6$ & $0.850 \pm 0.035-6$ \\
\hline 465 & $0.144 \pm 0.006-7$ & $0.610 \pm 0.025-7$ & $0.156 \pm 0.006-6$ & $0.283 \pm 0.011-6$ & $0.105 \pm 0.003-5$ \\
\hline 480 & $0.176 \pm 0.007-7$ & $0.844 \pm 0.029-7$ & $0.182 \pm 0.006-6$ & $0.353 \pm 0.013-6$ & $0.123 \pm 0.004-5$ \\
\hline 495 & $0.233 \pm 0.007-7$ & $0.972 \pm 0.031-7$ & $0.218 \pm 0.007-6$ & $0.464 \pm 0.014-6$ & $0.148 \pm 0.004-5$ \\
\hline 510 & $0.288 \pm 0.008-7$ & $0.122 \pm 0.003-6$ & $0.274 \pm 0.007-6$ & $0.583 \pm 0.016-6$ & $0.171 \pm 0.004-5$ \\
\hline 525 & $0.360 \pm 0.009-7$ & $0.143 \pm 0.003-6$ & $0.330 \pm 0.007-6$ & $0.639 \pm 0.015-6$ & $0.205 \pm 0.004-5$ \\
\hline 540 & $0.455 \pm 0.010-7$ & $0.167 \pm 0.003-6$ & $0.392 \pm 0.008-6$ & $0.772 \pm 0.017-6$ & $0.238 \pm 0.005-5$ \\
\hline 555 & $0.549 \pm 0.011-7$ & $0.198 \pm 0.003-6$ & $0.458 \pm 0.009-6$ & $0.880 \pm 0.018-6$ & $0.278 \pm 0.005-5$ \\
\hline 570 & $0.700 \pm 0.028-7$ & $0.245 \pm 0.007-6$ & $0.513 \pm 0.021-6$ & $0.989 \pm 0.039-6$ & $0.310 \pm 0.011-5$ \\
\hline 585 & $0.867 \pm 0.031-7$ & $0.281 \pm 0.008-6$ & $0.573 \pm 0.022-6$ & $0.115 \pm 0.004-5$ & $0.363 \pm 0.012-5$ \\
\hline 600 & $0.102 \pm 0.003-6$ & $0.314 \pm 0.008-6$ & $0.687 \pm 0.024-6$ & $0.135 \pm 0.004-5$ & $0.386 \pm 0.012-5$ \\
\hline 615 & $0.118 \pm 0.003-6$ & $0.361 \pm 0.008-6$ & $0.724 \pm 0.024-6$ & $0.146 \pm 0.004-5$ & $0.427 \pm 0.012-5$ \\
\hline 630 & $0.137 \pm 0.003-6$ & $0.365 \pm 0.009-6$ & $0.740 \pm 0.024-6$ & $0.154 \pm 0.004-5$ & $0.487 \pm 0.013-5$ \\
\hline 645 & $0.142 \pm 0.003-6$ & $0.416 \pm 0.008-6$ & $0.849 \pm 0.022-6$ & $0.170 \pm 0.004-5$ & $0.500 \pm 0.012-5$ \\
\hline 660 & $0.155 \pm 0.003-6$ & $0.421 \pm 0.008-6$ & $0.879 \pm 0.022-6$ & $0.175 \pm 0.004-5$ & $0.528 \pm 0.012-5$ \\
\hline 675 & $0.160 \pm 0.003-6$ & $0.441 \pm 0.008-6$ & $0.990 \pm 0.023-6$ & $0.170 \pm 0.004-5$ & $0.550 \pm 0.012-5$ \\
\hline 690 & $0.173 \pm 0.007-6$ & $0.455 \pm 0.013-6$ & $0.979 \pm 0.038-6$ & $0.197 \pm 0.008-5$ & $0.609 \pm 0.024-5$ \\
\hline 705 & $0.170 \pm 0.006-6$ & $0.461 \pm 0.013-6$ & $0.102 \pm 0.003-5$ & $0.213 \pm 0.008-5$ & $0.653 \pm 0.025-5$ \\
\hline 720 & $0.175 \pm 0.006-6$ & $0.494 \pm 0.013-6$ & $0.107 \pm 0.003-5$ & $0.227 \pm 0.008-5$ & $0.634 \pm 0.023-5$ \\
\hline 735 & $0.169 \pm 0.006-6$ & $0.512 \pm 0.014-6$ & $0.102 \pm 0.003-5$ & $0.207 \pm 0.008-5$ & $0.660 \pm 0.024-5$ \\
\hline 750 & $0.180 \pm 0.003-6$ & $0.520 \pm 0.011-6$ & $0.105 \pm 0.002-5$ & $0.213 \pm 0.006-5$ & $0.672 \pm 0.019-5$ \\
\hline 765 & $0.178 \pm 0.003-6$ & $0.517 \pm 0.010-6$ & $0.110 \pm 0.002-5$ & $0.229 \pm 0.006-5$ & $0.701 \pm 0.018-5$ \\
\hline 780 & $0.167 \pm 0.003-6$ & $0.517 \pm 0.010-6$ & $0.113 \pm 0.002-5$ & $0.221 \pm 0.006-5$ & $0.752 \pm 0.019-5$ \\
\hline 795 & $0.168 \pm 0.003-6$ & $0.525 \pm 0.015-6$ & $0.113 \pm 0.004-5$ & $0.221 \pm 0.007-5$ & $0.767 \pm 0.028-5$ \\
\hline 810 & $0.174 \pm 0.003-6$ & $0.533 \pm 0.015-6$ & $0.117 \pm 0.004-5$ & $0.238 \pm 0.008-5$ & $0.727 \pm 0.027-5$ \\
\hline 825 & $0.174 \pm 0.003-6$ & $0.543 \pm 0.015-6$ & $0.117 \pm 0.004-5$ & $0.233 \pm 0.007-5$ & $0.840 \pm 0.029-5$ \\
\hline 840 & $0.167 \pm 0.003-6$ & $0.529 \pm 0.015-6$ & $0.120 \pm 0.004-5$ & $0.268 \pm 0.008-5$ & $0.812 \pm 0.028-5$ \\
\hline 855 & $0.168 \pm 0.003-6$ & $0.522 \pm 0.015-6$ & $0.126 \pm 0.004-5$ & $0.251 \pm 0.008-5$ & $0.794 \pm 0.028-5$ \\
\hline 870 & $0.168 \pm 0.003-6$ & $0.558 \pm 0.016-6$ & $0.118 \pm 0.004-5$ & $0.261 \pm 0.008-5$ & $0.809 \pm 0.028-5$ \\
\hline 885 & $0.173 \pm 0.003-6$ & $0.587 \pm 0.016-6$ & $0.129 \pm 0.004-5$ & $0.259 \pm 0.008-5$ & $0.818 \pm 0.029-5$ \\
\hline
\end{tabular}


of a more appropriate variable. The number of nucleons in the central-density region is proportional to $A$, while the number in the surface is proportional to $A^{2 / 3}$. In the local density approximation, one can expect that the surface nucleons give a contribution to the cross section per nucleon that decreases like $A^{-1 / 3}$. Plotting the data as a function of $A^{-1 / 3}$ gives the result shown in Fig. 7 .

As a function of $A^{-1 / 3}$, the cross section per nucleon is

TABLE V. Cross sections at $E=3.595 \mathrm{GeV}$ and $\theta=25.01^{\circ}$ as a function of energy loss $\omega(\mathrm{MeV})$. The notation $0.123 \pm 0.456-7$ stands for $(0.123 \pm 0.456) \times 10^{-7} \mathrm{mb} / \mathrm{sr} \mathrm{MeV}$.

\begin{tabular}{|c|c|c|c|c|c|}
\hline$\omega$ & ${ }^{4} \mathrm{He}$ & ${ }^{12} \mathrm{C}$ & ${ }^{27} \mathrm{Al}$ & ${ }^{56} \mathrm{Fe}$ & ${ }^{197} \mathrm{Au}$ \\
\hline 360 & & $0.299 \pm 0.139-10$ & $0.883 \pm 0.386-10$ & $0.119 \pm 0.059-9$ & \\
\hline 375 & & & $0.143 \pm 0.045-9$ & $0.142 \pm 0.056-9$ & $0.866 \pm 0.431-9$ \\
\hline 390 & & $0.437 \pm 0.159-10$ & $0.162 \pm 0.046-9$ & $0.149 \pm 0.058-9$ & \\
\hline 405 & $0.987 \pm 0.311-11$ & $0.706 \pm 0.183-10$ & $0.237 \pm 0.055-9$ & $0.321 \pm 0.092-9$ & $0.184 \pm 0.043-8$ \\
\hline 420 & & $0.802 \pm 0.185-10$ & $0.279 \pm 0.061-9$ & $0.604 \pm 0.114-9$ & $0.254 \pm 0.052-8$ \\
\hline 435 & $0.195 \pm 0.057-10$ & $0.135 \pm 0.024-9$ & $0.279 \pm 0.057-9$ & $0.668 \pm 0.115-9$ & $0.319 \pm 0.058-8$ \\
\hline 450 & $0.235 \pm 0.078-10$ & $0.159 \pm 0.026-9$ & $0.432 \pm 0.071-9$ & $0.123 \pm 0.015-8$ & $0.326 \pm 0.061-8$ \\
\hline 465 & $0.245 \pm 0.099-10$ & $0.188 \pm 0.028-9$ & $0.621 \pm 0.089-9$ & $0.996 \pm 0.141-9$ & $0.379 \pm 0.064-8$ \\
\hline 480 & $0.648 \pm 0.104-10$ & $0.266 \pm 0.029-9$ & $0.777 \pm 0.086-9$ & $0.173 \pm 0.017-8$ & $0.576 \pm 0.071-8$ \\
\hline 495 & $0.914 \pm 0.111-10$ & $0.438 \pm 0.039-9$ & $0.987 \pm 0.097-9$ & $0.193 \pm 0.018-8$ & $0.607 \pm 0.072-8$ \\
\hline 510 & $0.855 \pm 0.131-10$ & $0.507 \pm 0.042-9$ & $0.134 \pm 0.011-8$ & $0.312 \pm 0.024-8$ & $0.840 \pm 0.087-8$ \\
\hline 525 & $0.138 \pm 0.015-9$ & $0.635 \pm 0.048-9$ & $0.141 \pm 0.011-8$ & $0.371 \pm 0.026-8$ & $0.111 \pm 0.010-7$ \\
\hline 540 & $0.232 \pm 0.036-9$ & $0.893 \pm 0.116-9$ & $0.226 \pm 0.029-8$ & $0.419 \pm 0.068-8$ & $0.150 \pm 0.028-7$ \\
\hline 555 & $0.239 \pm 0.038-9$ & $0.117 \pm 0.012-8$ & $0.247 \pm 0.028-8$ & $0.582 \pm 0.080-8$ & $0.237 \pm 0.035-7$ \\
\hline 570 & $0.269 \pm 0.047-9$ & $0.159 \pm 0.014-8$ & $0.287 \pm 0.029-8$ & $0.795 \pm 0.090-8$ & $0.179 \pm 0.030-7$ \\
\hline 585 & $0.376 \pm 0.047-9$ & $0.186 \pm 0.015-8$ & $0.384 \pm 0.034-8$ & $0.892 \pm 0.093-8$ & $0.275 \pm 0.039-7$ \\
\hline 600 & $0.417 \pm 0.051-9$ & $0.204 \pm 0.016-8$ & $0.532 \pm 0.042-8$ & $0.947 \pm 0.101-8$ & $0.275 \pm 0.039-7$ \\
\hline 615 & $0.566 \pm 0.046-9$ & $0.234 \pm 0.015-8$ & $0.502 \pm 0.036-8$ & $0.100 \pm 0.009-7$ & $0.376 \pm 0.036-7$ \\
\hline 630 & $0.690 \pm 0.049-9$ & $0.306 \pm 0.017-8$ & $0.692 \pm 0.042-8$ & $0.148 \pm 0.011-7$ & $0.430 \pm 0.038-7$ \\
\hline 645 & $0.846 \pm 0.076-9$ & $0.320 \pm 0.027-8$ & $0.831 \pm 0.080-8$ & $0.171 \pm 0.023-7$ & $0.530 \pm 0.065-7$ \\
\hline 660 & $0.117 \pm 0.008-8$ & $0.468 \pm 0.034-8$ & $0.108 \pm 0.009-7$ & $0.191 \pm 0.025-7$ & $0.712 \pm 0.078-7$ \\
\hline 675 & $0.130 \pm 0.009-8$ & $0.586 \pm 0.037-8$ & $0.145 \pm 0.010-7$ & $0.252 \pm 0.028-7$ & $0.683 \pm 0.074-7$ \\
\hline 690 & $0.158 \pm 0.010-8$ & $0.660 \pm 0.039-8$ & $0.165 \pm 0.011-7$ & $0.294 \pm 0.030-7$ & $0.986 \pm 0.088-7$ \\
\hline 705 & $0.197 \pm 0.011-8$ & $0.826 \pm 0.044-8$ & $0.201 \pm 0.012-7$ & $0.403 \pm 0.036-7$ & $0.126 \pm 0.010-6$ \\
\hline 720 & $0.238 \pm 0.010-8$ & $0.106 \pm 0.004-7$ & $0.252 \pm 0.011-7$ & $0.517 \pm 0.032-7$ & $0.166 \pm 0.009-6$ \\
\hline 735 & $0.304 \pm 0.012-8$ & $0.132 \pm 0.004-7$ & $0.295 \pm 0.011-7$ & $0.618 \pm 0.035-7$ & $0.177 \pm 0.009-6$ \\
\hline 750 & $0.351 \pm 0.013-8$ & $0.157 \pm 0.005-7$ & $0.355 \pm 0.013-7$ & $0.728 \pm 0.037-7$ & $0.216 \pm 0.010-6$ \\
\hline 765 & $0.499 \pm 0.028-8$ & $0.185 \pm 0.009-7$ & $0.424 \pm 0.021-7$ & $0.841 \pm 0.060-7$ & $0.303 \pm 0.019-6$ \\
\hline 780 & $0.601 \pm 0.030-8$ & $0.224 \pm 0.010-7$ & $0.566 \pm 0.024-7$ & $0.108 \pm 0.006-6$ & $0.311 \pm 0.020-6$ \\
\hline 795 & $0.674 \pm 0.032-8$ & $0.267 \pm 0.011-7$ & $0.655 \pm 0.026-7$ & $0.126 \pm 0.007-6$ & $0.331 \pm 0.020-6$ \\
\hline 810 & $0.779 \pm 0.036-8$ & $0.340 \pm 0.013-7$ & $0.740 \pm 0.028-7$ & $0.134 \pm 0.007-6$ & $0.424 \pm 0.023-6$ \\
\hline 825 & $0.989 \pm 0.039-8$ & $0.350 \pm 0.013-7$ & $0.757 \pm 0.028-7$ & $0.157 \pm 0.008-6$ & $0.497 \pm 0.025-6$ \\
\hline 840 & $0.118 \pm 0.003-7$ & $0.422 \pm 0.011-7$ & $0.870 \pm 0.025-7$ & $0.171 \pm 0.006-6$ & $0.521 \pm 0.019-6$ \\
\hline 855 & $0.136 \pm 0.004-7$ & $0.472 \pm 0.012-7$ & $0.962 \pm 0.026-7$ & $0.193 \pm 0.006-6$ & $0.620 \pm 0.021-6$ \\
\hline 870 & $0.159 \pm 0.008-7$ & $0.521 \pm 0.019-7$ & $0.112 \pm 0.004-6$ & $0.230 \pm 0.009-6$ & $0.679 \pm 0.033-6$ \\
\hline 885 & $0.183 \pm 0.009-7$ & $0.539 \pm 0.019-7$ & $0.127 \pm 0.004-6$ & $0.235 \pm 0.009-6$ & $0.783 \pm 0.036-6$ \\
\hline 900 & $0.207 \pm 0.009-7$ & $0.641 \pm 0.021-7$ & $0.135 \pm 0.005-6$ & $0.256 \pm 0.009-6$ & $0.839 \pm 0.037-6$ \\
\hline 915 & $0.224 \pm 0.010-7$ & $0.719 \pm 0.022-7$ & $0.152 \pm 0.005-6$ & $0.285 \pm 0.010-6$ & $0.894 \pm 0.038-6$ \\
\hline 930 & $0.279 \pm 0.010-7$ & $0.713 \pm 0.022-7$ & $0.152 \pm 0.005-6$ & $0.307 \pm 0.010-6$ & $0.937 \pm 0.039-6$ \\
\hline 945 & $0.257 \pm 0.007-7$ & $0.774 \pm 0.018-7$ & $0.167 \pm 0.004-6$ & $0.307 \pm 0.008-6$ & $0.103 \pm 0.002-5$ \\
\hline 960 & $0.304 \pm 0.008-7$ & $0.840 \pm 0.018-7$ & $0.171 \pm 0.004-6$ & $0.328 \pm 0.008-6$ & $0.100 \pm 0.002-5$ \\
\hline 975 & $0.329 \pm 0.011-7$ & $0.871 \pm 0.029-7$ & $0.198 \pm 0.007-6$ & $0.375 \pm 0.014-6$ & $0.110 \pm 0.003-5$ \\
\hline 990 & $0.335 \pm 0.012-7$ & $0.929 \pm 0.030-7$ & $0.186 \pm 0.007-6$ & $0.360 \pm 0.014-6$ & $0.124 \pm 0.004-5$ \\
\hline 1005 & $0.329 \pm 0.012-7$ & $0.101 \pm 0.003-6$ & $0.201 \pm 0.008-6$ & $0.413 \pm 0.015-6$ & $0.118 \pm 0.004-5$ \\
\hline 1020 & $0.355 \pm 0.012-7$ & $0.936 \pm 0.029-7$ & $0.206 \pm 0.007-6$ & $0.419 \pm 0.015-6$ & $0.125 \pm 0.004-5$ \\
\hline 1035 & $0.352 \pm 0.008-7$ & $0.102 \pm 0.003-6$ & $0.222 \pm 0.008-6$ & $0.398 \pm 0.015-6$ & $0.129 \pm 0.004-5$ \\
\hline 1050 & $0.358 \pm 0.007-7$ & $0.101 \pm 0.002-6$ & $0.228 \pm 0.006-6$ & $0.425 \pm 0.012-6$ & $0.137 \pm 0.003-5$ \\
\hline 1065 & $0.360 \pm 0.007-7$ & $0.105 \pm 0.002-6$ & $0.230 \pm 0.005-6$ & $0.485 \pm 0.013-6$ & $0.138 \pm 0.003-5$ \\
\hline 1080 & $0.378 \pm 0.009-7$ & $0.113 \pm 0.003-6$ & $0.246 \pm 0.008-6$ & $0.518 \pm 0.020-6$ & $0.153 \pm 0.005-5$ \\
\hline 1095 & $0.378 \pm 0.009-7$ & $0.114 \pm 0.003-6$ & $0.260 \pm 0.008-6$ & $0.495 \pm 0.020-6$ & $0.155 \pm 0.005-5$ \\
\hline 1110 & $0.375 \pm 0.009-7$ & $0.115 \pm 0.003-6$ & $0.257 \pm 0.008-6$ & $0.521 \pm 0.021-6$ & $0.156 \pm 0.005-5$ \\
\hline 1125 & $0.394 \pm 0.009-7$ & $0.122 \pm 0.003-6$ & $0.269 \pm 0.008-6$ & $0.519 \pm 0.021-6$ & $0.167 \pm 0.005-5$ \\
\hline 1140 & $0.404 \pm 0.010-7$ & $0.119 \pm 0.003-6$ & $0.280 \pm 0.008-6$ & $0.508 \pm 0.020-6$ & $0.175 \pm 0.006-5$ \\
\hline 1155 & $0.415 \pm 0.010-7$ & $0.130 \pm 0.003-6$ & $0.285 \pm 0.009-6$ & $0.603 \pm 0.022-6$ & $0.185 \pm 0.006-5$ \\
\hline
\end{tabular}


linear (with the exception of ${ }^{4} \mathrm{He}$ for which the assumptions needed to apply the local density approximation are not valid), and can easily be extrapolated to the nuclear matter cross section, i.e., $A^{-1 / 3}=0$. We have shown in a previous paper [5] that this linear dependence on $A^{-1 / 3}$ is valid for all $\mathbf{q}, \omega$ covered by this experiment. We have therefore concluded that the $A$ dependence of the inclusive cross section per nucleon is qualitatively understood. We note that the same $A^{-1 / 3}$ dependence is also valid in the DIS region, as shown by a recent analysis of the

TABLE VI. Cross sections at $E=3.595 \mathrm{GeV}$ and $\theta=30.01^{\circ}$ as a function of energy loss $\omega(\mathrm{MeV})$. The notation $0.123 \pm 0.456-7$ stands for $(0.123 \pm 0.456) \times 10^{-7} \mathrm{mb} / \mathrm{sr} \mathrm{MeV}$.

\begin{tabular}{|c|c|c|c|c|c|}
\hline$\omega$ & ${ }^{4} \mathrm{He}$ & ${ }^{12} \mathrm{C}$ & ${ }^{27} \mathrm{Al}$ & ${ }^{56} \mathrm{Fe}$ & ${ }^{197} \mathrm{Au}$ \\
\hline 600 & & & & $0.925 \pm 0.453-10$ & \\
\hline 630 & $0.630 \pm 0.187-11$ & & & & \\
\hline 645 & $0.629 \pm 0.139-11$ & & & $0.122 \pm 0.036-9$ & \\
\hline 660 & $0.385 \pm 0.126-11$ & & & $0.199 \pm 0.041-9$ & \\
\hline 675 & $0.378 \pm 0.154-11$ & & & $0.220 \pm 0.040-9$ & \\
\hline 690 & $0.873 \pm 0.195-11$ & & & $0.297 \pm 0.049-9$ & \\
\hline 705 & $0.170 \pm 0.039-10$ & & & $0.282 \pm 0.069-9$ & \\
\hline 720 & $0.129 \pm 0.035-10$ & & & $0.352 \pm 0.077-9$ & \\
\hline 735 & $0.168 \pm 0.040-10$ & & & $0.544 \pm 0.102-9$ & \\
\hline 750 & $0.243 \pm 0.039-10$ & & & $0.519 \pm 0.073-9$ & \\
\hline 765 & $0.341 \pm 0.043-10$ & & & $0.700 \pm 0.083-9$ & \\
\hline 780 & $0.485 \pm 0.049-10$ & & & $0.827 \pm 0.093-9$ & \\
\hline 795 & $0.476 \pm 0.083-10$ & & & $0.119 \pm 0.016-8$ & \\
\hline 810 & $0.617 \pm 0.100-10$ & & & $0.123 \pm 0.016-8$ & \\
\hline 825 & $0.889 \pm 0.107-10$ & & & $0.151 \pm 0.018-8$ & \\
\hline 840 & $0.102 \pm 0.011-9$ & & & $0.177 \pm 0.019-8$ & \\
\hline 855 & $0.105 \pm 0.013-9$ & & & $0.197 \pm 0.020-8$ & \\
\hline 870 & $0.151 \pm 0.013-9$ & & & $0.291 \pm 0.023-8$ & \\
\hline 885 & $0.153 \pm 0.013-9$ & & & $0.324 \pm 0.025-8$ & \\
\hline 900 & $0.258 \pm 0.034-9$ & & & $0.324 \pm 0.053-8$ & \\
\hline 915 & $0.244 \pm 0.034-9$ & & & $0.614 \pm 0.076-8$ & \\
\hline 930 & $0.336 \pm 0.037-9$ & & & $0.624 \pm 0.073-8$ & \\
\hline 945 & $0.342 \pm 0.038-9$ & & & $0.705 \pm 0.079-8$ & \\
\hline 960 & $0.414 \pm 0.042-9$ & & & $0.992 \pm 0.096-8$ & \\
\hline 975 & $0.589 \pm 0.038-9$ & $0.267 \pm 0.029-8$ & $0.567 \pm 0.089-8$ & $0.100 \pm 0.008-7$ & $0.417 \pm 0.055-7$ \\
\hline 990 & $0.703 \pm 0.045-9$ & $0.254 \pm 0.027-8$ & $0.685 \pm 0.092-8$ & $0.122 \pm 0.009-7$ & $0.487 \pm 0.056-7$ \\
\hline 1005 & $0.801 \pm 0.071-9$ & $0.283 \pm 0.027-8$ & $0.703 \pm 0.091-8$ & $0.169 \pm 0.019-7$ & $0.448 \pm 0.052-7$ \\
\hline 1020 & $0.101 \pm 0.008-8$ & $0.367 \pm 0.032-8$ & $0.100 \pm 0.011-7$ & $0.191 \pm 0.021-7$ & $0.620 \pm 0.065-7$ \\
\hline 1035 & $0.128 \pm 0.009-8$ & $0.506 \pm 0.038-8$ & $0.957 \pm 0.111-8$ & $0.272 \pm 0.025-7$ & $0.639 \pm 0.065-7$ \\
\hline 1050 & $0.155 \pm 0.009-8$ & $0.543 \pm 0.038-8$ & $0.117 \pm 0.011-7$ & $0.223 \pm 0.021-7$ & $0.668 \pm 0.061-7$ \\
\hline 1065 & $0.198 \pm 0.010-8$ & $0.646 \pm 0.042-8$ & $0.151 \pm 0.013-7$ & $0.274 \pm 0.025-7$ & $0.873 \pm 0.074-7$ \\
\hline 1080 & $0.222 \pm 0.008-8$ & $0.720 \pm 0.029-8$ & $0.172 \pm 0.008-7$ & $0.318 \pm 0.018-7$ & $0.970 \pm 0.049-7$ \\
\hline 1095 & $0.275 \pm 0.009-8$ & $0.834 \pm 0.030-8$ & $0.189 \pm 0.009-7$ & $0.395 \pm 0.020-7$ & $0.118 \pm 0.005-6$ \\
\hline 1110 & $0.325 \pm 0.014-8$ & $0.954 \pm 0.041-8$ & $0.235 \pm 0.011-7$ & $0.406 \pm 0.026-7$ & $0.133 \pm 0.007-6$ \\
\hline 1125 & $0.365 \pm 0.016-8$ & $0.112 \pm 0.004-7$ & $0.268 \pm 0.012-7$ & $0.502 \pm 0.029-7$ & $0.138 \pm 0.007-6$ \\
\hline 1140 & $0.437 \pm 0.017-8$ & $0.121 \pm 0.004-7$ & $0.283 \pm 0.013-7$ & $0.533 \pm 0.030-7$ & $0.177 \pm 0.008-6$ \\
\hline 1155 & $0.510 \pm 0.018-8$ & $0.131 \pm 0.005-7$ & $0.284 \pm 0.013-7$ & $0.608 \pm 0.032-7$ & $0.172 \pm 0.008-6$ \\
\hline 1170 & $0.523 \pm 0.017-8$ & $0.150 \pm 0.004-7$ & $0.325 \pm 0.010-7$ & $0.643 \pm 0.026-7$ & $0.194 \pm 0.006-6$ \\
\hline 1185 & $0.619 \pm 0.018-8$ & $0.167 \pm 0.004-7$ & $0.367 \pm 0.011-7$ & $0.717 \pm 0.028-7$ & $0.214 \pm 0.006-6$ \\
\hline 1200 & $0.715 \pm 0.044-8$ & $0.180 \pm 0.007-7$ & $0.342 \pm 0.016-7$ & $0.769 \pm 0.046-7$ & $0.226 \pm 0.010-6$ \\
\hline 1215 & $0.779 \pm 0.044-8$ & $0.194 \pm 0.007-7$ & $0.416 \pm 0.017-7$ & $0.867 \pm 0.048-7$ & $0.255 \pm 0.010-6$ \\
\hline 1230 & $0.746 \pm 0.042-8$ & $0.226 \pm 0.007-7$ & $0.408 \pm 0.017-7$ & $0.834 \pm 0.046-7$ & $0.256 \pm 0.010-6$ \\
\hline 1245 & $0.852 \pm 0.045-8$ & $0.216 \pm 0.007-7$ & $0.509 \pm 0.019-.7$ & $0.896 \pm 0.047-7$ & $0.267 \pm 0.010-6$ \\
\hline 1260 & $0.917 \pm 0.032-8$ & $0.231 \pm 0.006-7$ & $0.543 \pm 0.017-7$ & $0.993 \pm 0.040-7$ & $0.295 \pm 0.010-6$ \\
\hline 1275 & $0.994 \pm 0.033-8$ & $0.246 \pm 0.006-7$ & $0.533 \pm 0.016-7$ & $0.105 \pm 0.004-6$ & $0.335 \pm 0.010-6$ \\
\hline 1290 & $0.991 \pm 0.044-8$ & $0.284 \pm 0.011-7$ & $0.548 \pm 0.033-7$ & $0.113 \pm 0.006-6$ & $0.337 \pm 0.023-6$ \\
\hline 1305 & $0.963 \pm 0.047-8$ & $0.278 \pm 0.011-7$ & $0.550 \pm 0.033-7$ & $0.110 \pm 0.006-6$ & $0.384 \pm 0.024-6$ \\
\hline 1320 & $0.102 \pm 0.004-7$ & $0.292 \pm 0.011-7$ & $0.591 \pm 0.033-7$ & $0.119 \pm 0.006-6$ & $0.393 \pm 0.024-6$ \\
\hline 1335 & $0.101 \pm 0.004-7$ & $0.313 \pm 0.011-7$ & $0.678 \pm 0.036-7$ & $0.135 \pm 0.007-6$ & $0.412 \pm 0.024-6$ \\
\hline 1350 & $0.100 \pm 0.003-7$ & $0.321 \pm 0.009-7$ & $0.728 \pm 0.027-7$ & $0.136 \pm 0.006-6$ & $0.414 \pm 0.016-6$ \\
\hline 1365 & $0.118 \pm 0.003-7$ & $0.333 \pm 0.009-7$ & $0.696 \pm 0.026-7$ & $0.162 \pm 0.006-6$ & $0.455 \pm 0.017-6$ \\
\hline 1380 & $0.138 \pm 0.006-7$ & $0.339 \pm 0.013-7$ & $0.787 \pm 0.037-7$ & $0.155 \pm 0.010-6$ & $0.479 \pm 0.022-6$ \\
\hline 1395 & $0.123 \pm 0.005-7$ & $0.359 \pm 0.013-7$ & $0.802 \pm 0.038-7$ & $0.175 \pm 0.011-6$ & $0.480 \pm 0.022-6$ \\
\hline
\end{tabular}


TABLE VI. (Continued).

\begin{tabular}{cccccc}
\hline \hline$\omega$ & ${ }^{4} \mathrm{He}$ & ${ }^{12} \mathrm{C}$ & ${ }^{27} \mathrm{Al}$ & ${ }^{56} \mathrm{Fe}$ & ${ }^{197} \mathrm{Au}$ \\
\hline 1410 & $0.127 \pm 0.006-7$ & $0.372 \pm 0.013-7$ & $0.872 \pm 0.039-7$ & $0.179 \pm 0.011-6$ & $0.498 \pm 0.022-6$ \\
1425 & $0.138 \pm 0.006-7$ & $0.381 \pm 0.014-7$ & $0.874 \pm 0.039-7$ & $0.191 \pm 0.011-6$ & $0.613 \pm 0.025-6$ \\
1440 & $0.138 \pm 0.004-7$ & $0.434 \pm 0.011-7$ & $0.978 \pm 0.030-7$ & $0.176 \pm 0.006-6$ & $0.576 \pm 0.020-6$ \\
1455 & $0.152 \pm 0.004-7$ & $0.440 \pm 0.011-7$ & $0.103 \pm 0.003-6$ & $0.192 \pm 0.007-6$ & $0.594 \pm 0.020-6$ \\
1470 & $0.153 \pm 0.007-7$ & $0.465 \pm 0.016-7$ & $0.101 \pm 0.004-6$ & $0.206 \pm 0.009-6$ & $0.646 \pm 0.035-6$ \\
1485 & $0.171 \pm 0.007-7$ & $0.518 \pm 0.016-7$ & $0.106 \pm 0.004-6$ & $0.205 \pm 0.008-6$ & $0.653 \pm 0.035-6$ \\
1500 & $0.159 \pm 0.007-7$ & $0.497 \pm 0.016-7$ & $0.112 \pm 0.004-6$ & $0.210 \pm 0.008-6$ & $0.664 \pm 0.035-6$ \\
1515 & $0.182 \pm 0.005-7$ & $0.528 \pm 0.011-7$ & $0.119 \pm 0.003-6$ & $0.227 \pm 0.007-6$ & $0.710 \pm 0.025-6$ \\
\hline \hline
\end{tabular}

world data on ratios of DIS cross sections for nuclei to the deuteron as described in Ref. [17].

The differences between the response functions of various nuclei can be studied by plotting the ratio of the cross sections per nucleon, as was done for DIS in MEC-type experiments. In Fig. 8 we show the ratio of ${ }^{56} \mathrm{Fe} /{ }^{4} \mathrm{He}$, the nuclei for which our data cover the largest range of $\omega$. The ratio show a minimum at $x=1$, in accordance with the higher peak cross section of ${ }^{4} \mathrm{He}$ due to the smaller Fermi momentum. At large $x$ (low $\omega$ ) the ratio rises, and reaches a plateau at $x \sim 1.6$. Such a plateau has been predicted by the few-nucleon cluster model of Frankfurt and Strikman $[18,19]$. In this model, the plateau is a consequence of the fact that the tail of the momentum distribution at large $\mathbf{k}$ is similar in shape, but different in amplitude, for different nuclei.

TABLE VII. Cross sections at $E=3.995 \mathrm{GeV}$ and $\theta=30.05^{\circ}$ for ${ }^{56} \mathrm{Fe}$ as a function of energy loss $\omega(\mathrm{MeV})$. The notation $0.123 \pm 0.456-7$ stands for $(0.123 \pm 0.456) \times 10^{-7} \mathrm{mb} / \mathrm{sr} \mathrm{MeV}$.

\begin{tabular}{|c|c|c|c|c|c|}
\hline$\omega$ & $\sigma \pm \delta \sigma$ & $\omega$ & $\sigma \pm \delta \sigma$ & $\omega$ & $\sigma \pm \delta \sigma$ \\
\hline 825 & $0.634 \pm 0.304-10$ & 1350 & $0.256 \pm 0.010-7$ & 1875 & $0.197 \pm 0.007-6$ \\
\hline 840 & $0.657 \pm 0.303-10$ & 1365 & $0.268 \pm 0.010-7$ & 1890 & $0.205 \pm 0.007-6$ \\
\hline 855 & $0.993 \pm 0.310-10$ & 1380 & $0.297 \pm 0.010-7$ & 1905 & $0.210 \pm 0.008-6$ \\
\hline 870 & $0.964 \pm 0.306-10$ & 1395 & $0.329 \pm 0.009-7$ & 1920 & $0.206 \pm 0.008-6$ \\
\hline 885 & $0.102 \pm 0.030-9$ & 1410 & $0.354 \pm 0.009-7$ & 1935 & $0.224 \pm 0.006-6$ \\
\hline 900 & $0.102 \pm 0.030-9$ & 1425 & $0.380 \pm 0.016-7$ & 1950 & $0.234 \pm 0.009-6$ \\
\hline 915 & $0.192 \pm 0.033-9$ & 1440 & $0.412 \pm 0.015-7$ & 1965 & $0.243 \pm 0.009-6$ \\
\hline 930 & $0.222 \pm 0.034-9$ & 1455 & $0.432 \pm 0.015-7$ & 1980 & $0.239 \pm 0.009-6$ \\
\hline 945 & $0.280 \pm 0.050-9$ & 1470 & $0.478 \pm 0.017-7$ & 1995 & $0.260 \pm 0.007-6$ \\
\hline 960 & $0.338 \pm 0.044-9$ & 1485 & $0.509 \pm 0.018-7$ & 2010 & $0.272 \pm 0.007-6$ \\
\hline 975 & $0.362 \pm 0.046-9$ & 1500 & $0.535 \pm 0.014-7$ & 2025 & $0.289 \pm 0.007-6$ \\
\hline 990 & $0.550 \pm 0.081-9$ & 1515 & $0.543 \pm 0.014-7$ & 2040 & $0.291 \pm 0.010-6$ \\
\hline 1005 & $0.752 \pm 0.102-9$ & 1530 & $0.618 \pm 0.025-7$ & 2055 & $0.297 \pm 0.010-6$ \\
\hline 1020 & $0.647 \pm 0.090-9$ & 1545 & $0.653 \pm 0.026-7$ & 2070 & $0.306 \pm 0.010-6$ \\
\hline 1035 & $0.790 \pm 0.097-9$ & 1560 & $0.703 \pm 0.027-7$ & 2085 & $0.305 \pm 0.008-6$ \\
\hline 1050 & $0.102 \pm 0.010-8$ & 1575 & $0.718 \pm 0.021-7$ & 2100 & $0.328 \pm 0.012-6$ \\
\hline 1065 & $0.118 \pm 0.009-8$ & 1590 & $0.797 \pm 0.022-7$ & 2115 & $0.359 \pm 0.012-6$ \\
\hline 1080 & $0.137 \pm 0.009-8$ & 1605 & $0.819 \pm 0.022-7$ & 2130 & $0.335 \pm 0.012-6$ \\
\hline 1095 & $0.170 \pm 0.010-8$ & 1620 & $0.857 \pm 0.034-7$ & 2145 & $0.364 \pm 0.012-6$ \\
\hline 1110 & $0.211 \pm 0.016-8$ & 1635 & $0.830 \pm 0.034-7$ & 2160 & $0.375 \pm 0.009-6$ \\
\hline 1125 & $0.254 \pm 0.017-8$ & 1650 & $0.915 \pm 0.036-7$ & 2175 & $0.380 \pm 0.013-6$ \\
\hline 1140 & $0.284 \pm 0.018-8$ & 1665 & $0.958 \pm 0.028-7$ & 2190 & $0.364 \pm 0.012-6$ \\
\hline 1155 & $0.384 \pm 0.021-8$ & 1680 & $0.106 \pm 0.003-6$ & 2205 & $0.381 \pm 0.012-6$ \\
\hline 1170 & $0.425 \pm 0.020-8$ & 1695 & $0.115 \pm 0.004-6$ & 2220 & $0.407 \pm 0.013-6$ \\
\hline 1185 & $0.497 \pm 0.021-8$ & 1710 & $0.109 \pm 0.004-6$ & 2235 & $0.402 \pm 0.013-6$ \\
\hline 1200 & $0.600 \pm 0.024-8$ & 1725 & $0.113 \pm 0.004-6$ & & \\
\hline 1215 & $0.796 \pm 0.049-8$ & 1740 & $0.124 \pm 0.004-6$ & & \\
\hline 1230 & $0.920 \pm 0.052-8$ & 1755 & $0.131 \pm 0.004-6$ & & \\
\hline 1245 & $0.953 \pm 0.053-8$ & 1770 & $0.131 \pm 0.003-6$ & & \\
\hline 1260 & $0.115 \pm 0.005-7$ & 1785 & $0.145 \pm 0.006-6$ & & \\
\hline 1275 & $0.139 \pm 0.006-7$ & 1800 & $0.153 \pm 0.006-6$ & & \\
\hline 1290 & $0.151 \pm 0.005-7$ & 1815 & $0.155 \pm 0.006-6$ & & \\
\hline 1305 & $0.169 \pm 0.005-7$ & 1830 & $0.157 \pm 0.006-6$ & & \\
\hline 1320 & $0.202 \pm 0.009-7$ & 1845 & $0.172 \pm 0.005-6$ & & \\
\hline 1335 & $0.219 \pm 0.009-7$ & 1860 & $0.180 \pm 0.005-6$ & & \\
\hline
\end{tabular}




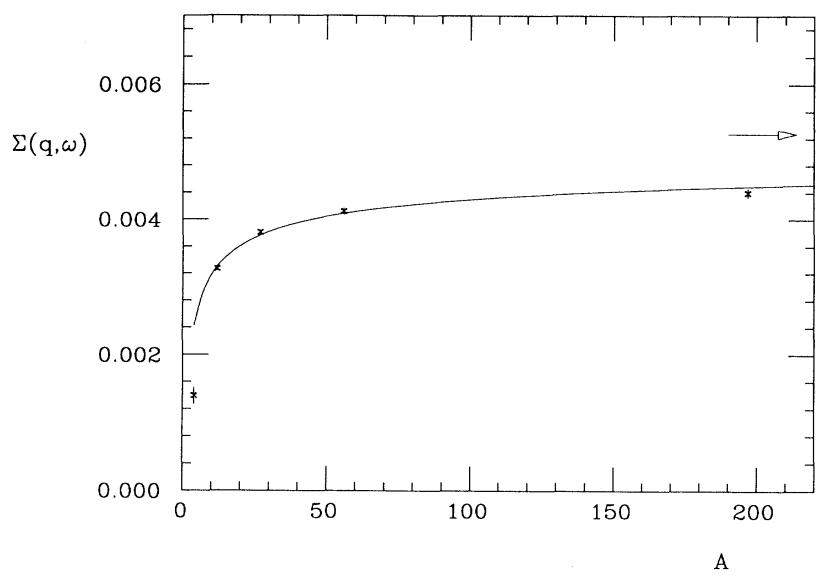

FIG. 6. Response function per nucleon at $E=3.595 \mathrm{GeV}$ and $\omega=180 \mathrm{MeV}$ as a function of $A$. The solid line is the fit of the response function in terms of $A^{-1 / 3}$. The arrow points to the value of the fit at $A=\infty$.

Other workers have also made predictions for cross section ratios. The calculation of Vary [20] is based on the quark cluster model. The contribution in the high- $x$ region from quarks in $3,6,9, \ldots$-quark clusters are calculated from the probability of finding such clusters and from the momentum distribution of the quarks in the cluster. The probability is calculated from the overlap of nucleons obtained from a nuclear wave function with uncorrelated nucleons, and the quark momentum distribution is obtained from naive quark counting rules. The resulting prediction for the cross section ratio is also shown in Fig. 8. The plateau in this model results from the sixquark cluster contribution.

The quantitative understanding of the region $\omega \leq \omega_{\text {qe }}$ in terms of nucleonic degrees of freedom requires a calculation based on a realistic spectral function $S(k, E)$.

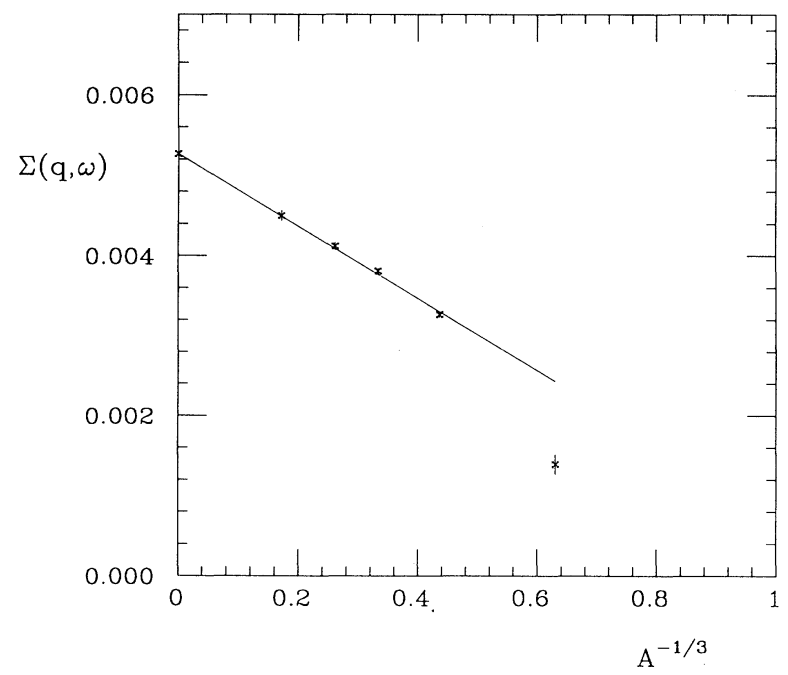

FIG. 7. Response function per nucleon as a function of $A^{-1 / 3}$ for $E=3.595 \mathrm{GeV}, \theta=16^{\circ}$, and $\omega=180 \mathrm{MeV}$. The solid line is the fit of the response function in terms of $A^{-1 / 3}$. Only data for $A \geq 12$ are used for the extrapolation to $A=\infty$.

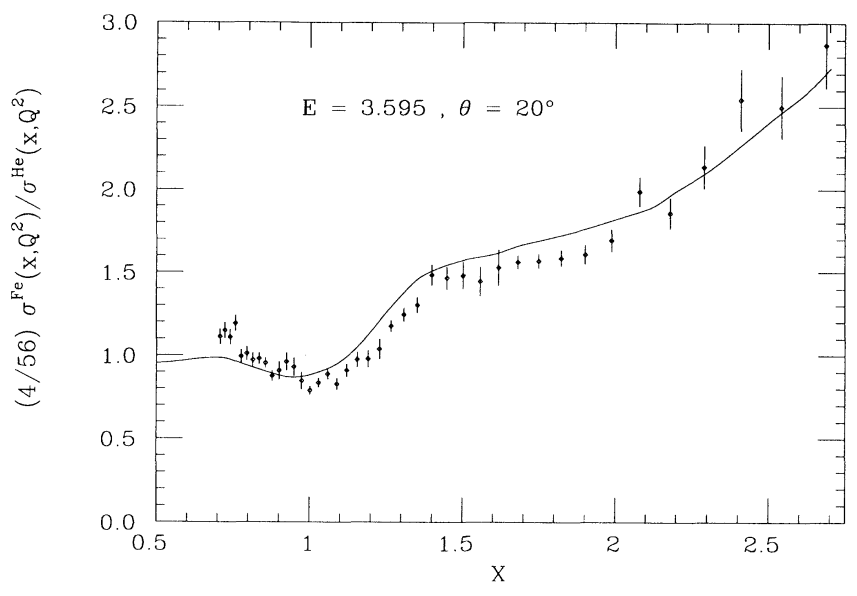

FIG. 8. Ratio of iron to helium cross sections as a function of energy loss, for $E=3.595 \mathrm{GeV}$, and $\theta=20^{\circ}$. The curve is from Ref. [20].

Such a calculation is presently available only for $A \leq 3$ and for nuclear matter. In order to be able to make the comparison of data and calculation quantitative, we have used the calculations for nuclear matter and the results obtained by extrapolating the finite nucleus data (presented in Tables I-VIII) to $A=\infty$.

The nuclear matter spectral function has been calculated by Benhar et al. [21]. This calculation is carried out within the framework of correlated basis function perturbation theory, using a complete set of orthonormal correlated many-body wave function states, generated by a realistic nuclear Hamiltonian involving two-body and three-body interactions.

The electron-nucleon cross section is calculated for an off-shell nucleon according to the approach of deForest [22]. This approach is extended to include excitation of nucleon resonances and DIS [7] by employing the inelastic response functions as parametrized by Bodek and Ritchie [15]. The nucleon final-state interaction is accounted for by using correlated Glauber theory for the recoiling nucleon. With this approach, developed in Ref. [7], it becomes possible to treat the final state quantitatively, and in a relativistically correct way. The effects of color transparency - the fact that immediately after a scattering process at large $\mathbf{q}$ the interaction of the recoiling nucleon is reduced due to its more pointlike configuration - are included as well.

In Fig. 9 we show the comparison between experimental and theoretical results for one energy and angle. Clearly, the theoretical curve agrees quantitatively with most of the features of the data. To achieve this accurate description of the cross sections it is important to use a spectral function with a realistic high-momentum tail as produced by short-range nucleon-nucleon correlations. It is also important to include the $N-N$ short-range correlations in the description of the final-state interaction. At low $\omega$ and very large $\mathbf{q}$, the cross sections are rather sensitive to the effects of color transparency.

Figure 9 shows that the region of nucleon resonances and DIS can be well understood. This calculation using 


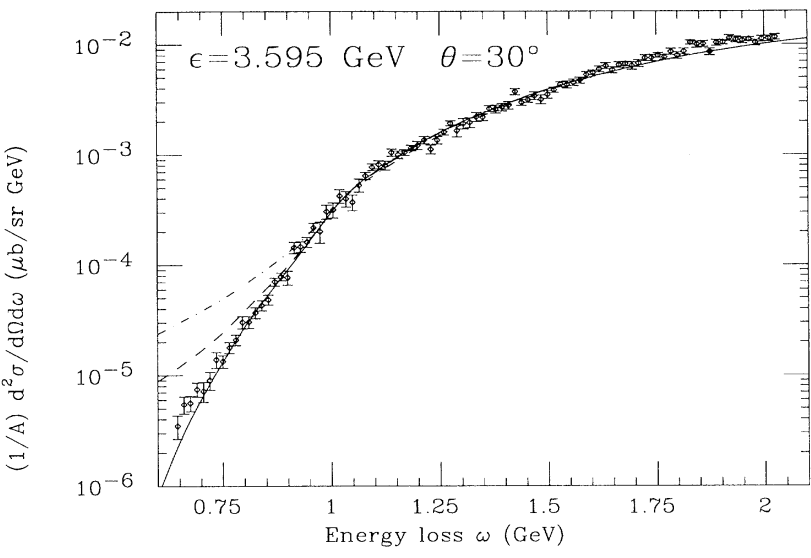

FIG. 9. Cross sections for $E=3.595 \mathrm{GeV}$ and $\theta=30^{\circ}$. The dot-dashed and dashed lines correspond to the results of [7] without and with short-range $N-N$ correlations. The solid curve includes the effects of color transparency.

a realistic spectral function and the measured proton and neutron inelastic response functions predicts the data accurately for $\omega \leq 1.5 \mathrm{GeV}$.

It is instructive to study the response of nuclei not only in terms of the cross section, but also in terms of the scaling function $F(y)$. The quasielastic inclusive response $a$ priori is a function of two independent variables $q$ and $\omega$. In the plane wave impulse approximation (PWIA) it can be shown that, as $q$ tends toward infinity, the cross sections will scale, i.e., become a function of a single variable $y$ which itself depends on $\mathbf{q}$ and $\omega$. The variable $y$ may be thought of as the smallest value of the momentum $\mathbf{k}$ of a nucleon bound with the smallest removal energy [3] allowed by momentum and energy conservation. In the limit $q=\infty$ the scaling function depends on $y$ only, and is related to the longitudinal momentum distribution $n\left(k_{\|}\right)$. This scaling has been shown to work very well for light nuclei $A \leq 3$.

In Fig. 10 we show the scaling function for ${ }^{4} \mathrm{He}$, with

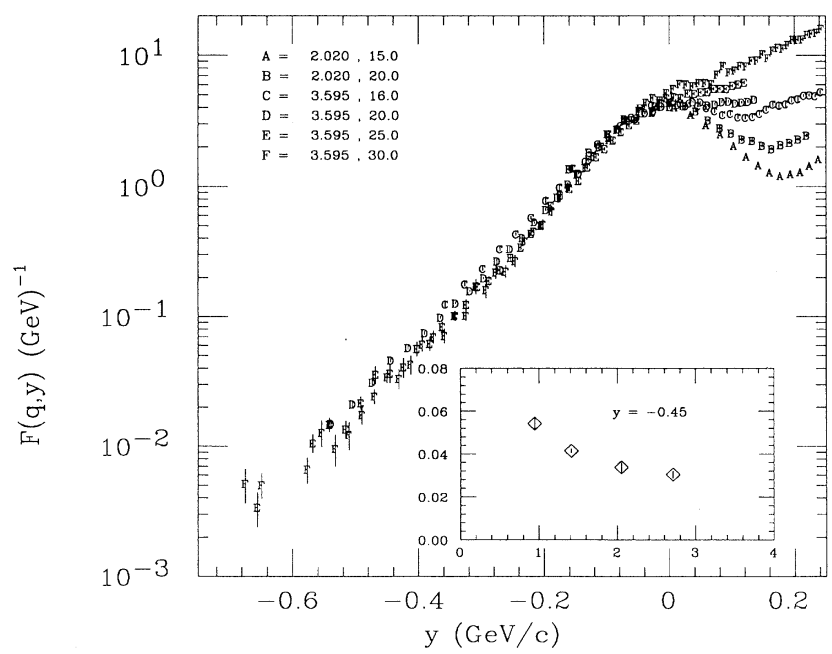

FIG. 10. The scaling function, $F(y)$, of ${ }^{4} \mathrm{He}$ obtained from the present experiment. The inset shows the convergence of $F(y)$ with $q$ at $y=-0.45 \mathrm{GeV} / c$.

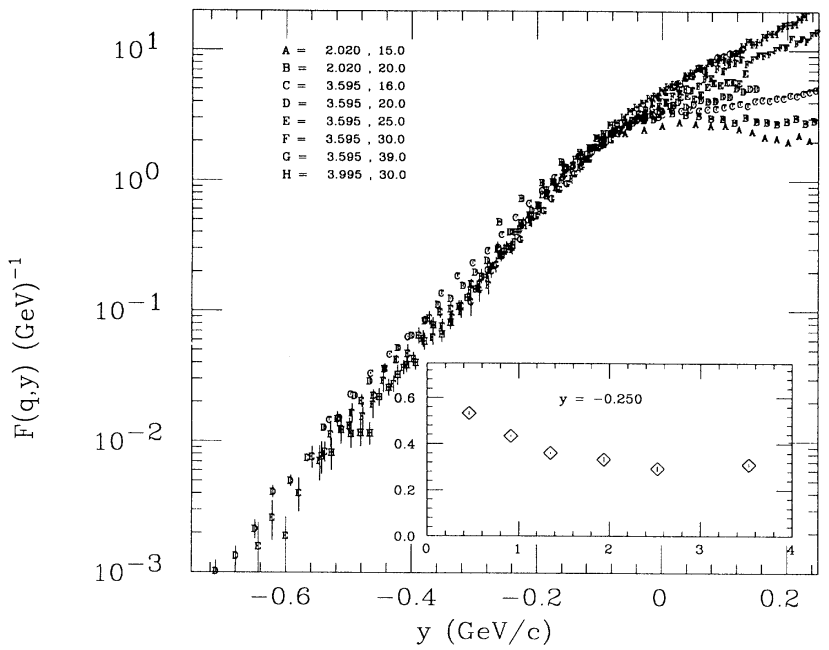

FIG. 11. The scaling function, $F(y)$, for the iron data. The inset shows the convergence of $F(y)$ with $q$ at $y=-0.25$ $\mathrm{GeV} / c$.

the corresponding scaling function for ${ }^{56} \mathrm{Fe}$ shown in Figure 11. The data do scale well for helium, whereas pronounced deviations are observed for ${ }^{56} \mathrm{Fe}$; these data converge more slowly to the asymptotic value of $F(y)$ at very large q. To a large degree this slower approach to scaling can be understood as a consequence of a deviation from PWIA. In a high-density nuclear medium, the recoiling nucleon is subject to final-state interaction (FSI) close to the point of interaction with the electron, and this part of FSI still influences the inclusive electron spectrum. The authors of Ref. [7] have studied the convergence of $F(y)$ for nuclear matter where the FSI can be calculated with a fair degree of accuracy. They have found that at large negative $y(-500 \mathrm{MeV} / c)$, FSI plays a major role while at low $y(-100 \mathrm{MeV} / c)$, where FSI are less important and the convergence is much faster.

This slower approach to scaling for heavy nuclei indicates that the reaction mechanism is not well described by PWIA; the complications due to FSI have to be accounted for before a quantitative understanding of the inclusive cross sections for finite $A$ can be achieved.

These data have also been studied in terms of the Nachtmann scaling variable $\xi$. This analysis [24] finds that at the highest $Q^{2}$ the data are consistent with the arguments of local duality.

\section{CONCLUSION}

In this paper we have presented data on inclusive electron scattering in the quasielastic region. These data cover a large region of momentum transfer $\mathbf{q}$. For the first time, such a study has been made systematically as a function of nuclear mass number, thus allowing access to the response functions of nuclear matter. Some of the physics contained in the data have been illustrated by comparison with a number of calculations in the previous section. Other theoretical work suggested by these 
data can be found in Refs. [27,23,25,26].

Extension of the data in several directions remains desirable.

With the present data it is not yet possible to study the region near $x=2$ in any detailed way - the statistical errors of the data are still too large. Such studies would be of high interest because one could identify eventual contributions from scattering systems heavier than the nucleon via scaling, such as the six-quark clusters mentioned above. To do so, one would have to extend the data to larger $x$, with lower statistical errors.

Separation of the cross sections into their longitudinal $(L)$ and transverse $(T)$ components would be of great interest. Such separations have been performed at low $\mathbf{q}, \sim 3 \mathrm{fm}^{-1}$, and have produced results that are not yet well understood. At large $\mathbf{q}$ the interpretation of the data would be easier as the sum rules usually applied are more valid. At large $\mathbf{q}$, the separation of the longitudinal response is more difficult [28], as the $L / T$ ratio decreases with increasing $\mathbf{q}$. With a dedicated experiment designed to produce accurate cross sections at large inelasticities, such a separation with the accuracy needed should be possible.

Further studies of the inclusive response are certainly needed, as they form the basis for the more exclusive cross sections now accessible with $\mathrm{cw}$ beams, and since they contain a wealth of information on both nucleonic and subnucleonic degrees of freedom.
[1] J. Aubert et al., Phys. Lett. B 123, 275 (1983).

[2] J. Ashman et al., Phys. Lett. B 206, 364 (1988).

[3] D. Day, J.S. McCarthy, T.W. Donnelly, and I. Sick, Annu. Rev. Nucl. Part. Sci. 40, 357 (1990).

[4] D. Day, J.S. McCarthy, Z.E. Meziani, R. Minehart, R. Sealock, S.T. Thornton, J. Jourdan, I. Sick, B.W. Filippone, R.D. McKeown, R.G. Milner, D.H. Potterveld, and Z. Szalata, Phys. Rev. Lett. 59, 427 (1987).

[5] D. Day, J.S. McCarthy, Z.E. Meziani, R. Minehart, R.M. Sealock, S. Thornton, J. Jourdan, I. Sick, B.W. Filippone, R.D. McKeown, R.G. Milner, D. Potterveld, and Z. Szalata, Phys. Rev. C 40, 1011 (1989).

[6] D.H. Potterveld, Ph.D. thesis, California Institute of Technology 1989.

[7] O. Benhar, A. Fabrocini, S. Fantoni, G.A. Miller, V.R. Pandharipande, and I. Sick, Phys. Rev. C 44, 2328 (1991).

[8] C. Ciofi degli Atti, D.B. Day, and S. Liuti, Phys. Rev. C 46, 1045 (1992).

[9] A Sill, Ph.D. thesis, American University.

[10] G.G. Simon, Ch. Schmitt, F. Borkowski, and V.H. Walther, Nucl. Phys. A 333381 (1980).

[11] S. Stein, W.B. Atwood, E.D. Bloom, R.L.A. Cottrell, H. DeStaebler, C.L. Jordan, H.G. Piel, C.Y. Prescott, R. Siemann, and R.E. Taylor, Phys. Rev. D 12, 1884 (1975).

[12] L.W. Mo and Y.S. Tsai, Rev. Mod. Phys. 46, 815 (1969).
[13] Y.S. Tsai, SLAC Report No. SLAC-PUB-848, 1971.

[14] D.B. Day, Nucl. Phys. A 532, 255c (1991).

[15] A. Bodek and J.L. Ritchie, Phys. Rev. D 23, 1070 (1981).

[16] T. de Forest, Jr., Nucl. Phys. A 132, 305 (1969).

[17] I. Sick and D.B. Day, Phys. Lett B 274, 16 (1992).

[18] L. Frankfurt and M. Strikman, Phys. Rep. 76, 215 (1981).

[19] L. Frankfurt and M. Strikman, Phys. Rep. 160, 235 (1988).

[20] J. Vary, in The Three-Body Force in the Three-Nucleon System, Proceedings of the International Symposium, Washington, D.C., 1986, edited by B.L. Berman and B.F. Gibson, Lecture Notes in Physics Vol. 260 (Springer, Berlin, 1986), p. 422.

[21] O. Benhar, A. Fabrocini, and S. Fantoni, Nucl. Phys. A 505, 267 (1989).

[22] A.E.L. Dieperink, T. de Forest, I. Sick, and R.A. Brandenburg, Phys. Lett. 63B, 261 (1976).

[23] X. Ji and J. Engel, Phys. Rev. C 40, 497 (1989).

[24] B.W. Filippone et al., Phys. Rev. C 45, 1582 (1992).

[25] X. Ji and B.W. Filippone, Phys. Rev. C 42, R2279 (1990).

[26] X. Ji and R.D. McKeown, Phys. Lett. B 236, 130 (1990).

[27] C. Carraro and S.E. Koonin, Nucl. Phys. A 524, 201 (1991).

[28] J.P. Chen et al., Phys. Rev. Lett. 66, 1283 (1991). 